\title{
COMBINED EFFECT OF SOME PLANT EXTRACTS UNDER MODIFIED ATMOSPHERE OF CARBON DIOXIDE AGAINST STORED GRAIN INSECTS
}

\author{
EL-LAKWAH, F.A. ${ }^{1}$, Z.A. HALAWA ${ }^{2}$ M.M. KHATTAB ${ }^{1}$ \\ AND T.A. ABDEL -RAHMAN ${ }^{1}$
}

\author{
1. Plant Protection Department, Faculty of Agriculture at Moshtohor, Zagazig \\ University \\ 2. Plant Protection Research Institute, ARC, Ministry of Agriculture, Cairo, Egypt.
}

(Manuscript received 3 November 2010 )

\begin{abstract}
Where, tests were conducted inside fiberglass bins during summer time, the temperature of grain was $32 \pm 2^{\circ} \mathrm{C}$. The plant extracts tested were the acetone and petroleum ether extracts of Poinciana seeds (Delonix regia); Cloves flowering buds (Syzgium aromaticum); Cinnamon (Cinnamonum zeylancicum); Radish seeds (Raphanus sativus); and Mustard seeds (Brassica alba). Test insects were Sitophilus orzae(L); Rhizopertha dominica (F.) and Tribolium Castaneum.

The various plant extracts were tested alone at $0.4 \%$ and $0.8 \%(\mathrm{w} / \mathrm{w})$ concentrations and under modified atmosphere (MA) of $20 \pm 5 \% \mathrm{CO}_{2}$. Results concerning Co-toxicity values resulted from addition of $20 \pm 5 \% \mathrm{CO}_{2}$ to the acetone and petroleum ether extracts of various plants revealed that the extracts at $0.4 \%$ and $0.8 \%$ produced additive effects for all tested insect species at all exposure periods. Comparing the toxicity of these materials to the insect species, the rice weevil Sitophilus oryzae (L.) was the most sensitive, followed by the lesser grain borer Rhizopertha dominica (F) and the red flour beetle Tribolium castaneum (Herbst) adults. Meanwhile, treatment of various insect species with the plant extracts under modified atmosphere of $\mathrm{CO}_{2}$ produced obviously higher mortality values than each treatment alone and proved to be effective against the three insect species under study.
\end{abstract}

\section{INTRODUCTION}

Today, attention has been focused to control stored product pests with other alternative pest control agents such as extracts of plant leaves, flowers and seeds as powders (Ivbijaro, 1984; Su, 1985; Halawa, 2003 and 2004; 2005 and Halawa et al., 2009).

Controlled or modified atmospheres (MA) received considerable attention in recent years as one of the alternative methods to control stored product insects.

The effectiveness of $\mathrm{Co}_{2}$ to some stored products insects is increased with reduced oxygen concentrations at least down to $7 \%$ level. But Sitophilus spp. had shown a reversal response at very low oxygen concentrations (Krishnamurthy et al., 1986 and Reichmuth, 1986). 
The exposure of insects to carbon dioxide and its effect on insect mortality were studied by some investigators (Harein and Press, 1968; Aliniazee, 1971; Jay and Pearman, 1971 and Tunc, 1983).

The aim of this study was to investigate the combined effect of the plant extracts and the MA of $20 \pm 5 \% \mathrm{Co}_{2}$ against certain insects infesting stored grains.

\section{MATERIALS AND METHODS}

\section{Insects:}

Laboratory strains of three stored products insects namely the rice weevil Sitophilus oryzae (L.) (Curculionidae, Coleopetera); the lesser grain borer Rhizopertha dominica (F.) (Bostrychidae) and the red flour beetle Tribolium castaneum (Herbst) were used during this study.

Insects were maintained at the stored products pests laboratory of the plant protection Dept., Faculty of Agric., Moshtohor, Zagazig University.

\section{Plant extracts:}

Seeds of Poinciana (Delonix regia), cloves flowering buds (Syzygium aromaticum), cinnamon (Cinnamonum zeylanicum), radish seeds (Raphanus sativus) and mustard seeds (Brassica alba) were either bought from the local market or from the farm of the faculty. The active ingredient present in the preceding plant species are:

1- Poinciana: L-Azetidine-2-carboxylic acid (Cromwell, 1979).

2- Cloves: Casuarictin and Eugeniin (Tellimagrandin II) (Okuda, 1983).

3- Cinnamon: Carpacin (Isosafrole methyl ether) (Mohandas, 1969).

4- Radish seeds: Brassinin (Shimizu, 1986) and (Methyl Mercaptan 9 Methane thiol: Methyl thioalcohol) (Keethaas, 1931).

5- Mustard seeds: S-Methyl-L-Cysteine S-oxide (3-"Methyl sulfonyl" alanine) (Bansley, 1968).

These plants were ground using a high speed mill into a fine powder and then extracted with acetone at $50^{\circ} \mathrm{C}$ under reduced pressure to a small volume as described by Su (1985). The crude gum of the extract was weighed and dissolved by the solvent to get $10 \%(\mathrm{w} / \mathrm{v})$ stock solution. Concentrations were prepared by diluting the stock solution using the same solvent. Five $\mathrm{ml}$ of the stock solution was added to fifty grams of maize grains to obtain a concentration of $1 \% \mathrm{w} / \mathrm{w}$ Grain was mixed well with and left for two hours for air dryness. Thirty adult insects were confined with the treated maize grains in the tests.

Three replicates were used for each concentration. Jars were covered with muslin and rubber band. Untreated grains were mixed only with $5 \mathrm{ml}$ solvent for complete 
coverage. Mortality was assessed after 1, 2, 3, 5, 7 and 14 days from treatment, jars were kept at $26 \pm 2{ }^{\circ} \mathrm{C}$ and $65 \pm 5 \%$ R.H.

Reduction in F1-progeny was calculated after 60 days from treatment according to the following equation:

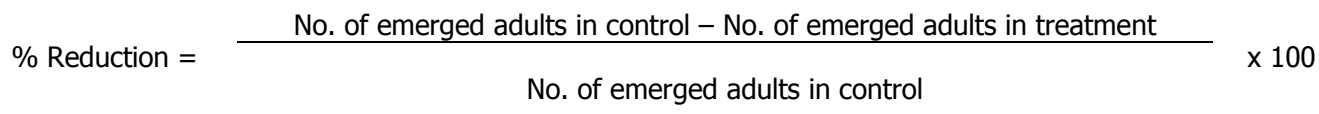

Tests of modified atmosphere (MA) of carbon dioxide were achieved inside closed fiberglass bins, each of $\mathrm{Ca} 0.2 \mathrm{~m}^{3}$ volume.

Each Fiberglass bin was provided with one inlet tube near its top and one outlet near the bottom for insersion of carbon dioxide as well as for monitoring of $\mathrm{CO}_{2}$ concentration inside the bin. The sealing of the bins was improved by constructing a well closed covers. The bins were kept under storage conditions inside a store-room during the whole period of the experiment.

\section{Technique:}

Bags, each containing $50 \mathrm{~g}$ of maize grains and 30 adults of the test insect were inserted inside the bins. The cover of each bin was closed well. Modified atmosphere of $25 \% \mathrm{Co}_{2}$ was established inside the bins.

After the desired exposure period, each glass bin was aerated and insects, were transferred to the laboratory for mortality assessment. Mortality data were corrected using Abbot's formula (1925). Grains temperature and the relative humidity inside the metal drum were recorded during the tests.

\section{Determination of $\mathrm{Co}_{2}$ concentration:}

Carbon dioxide $\left(\mathrm{CO}_{2}\right)$ concentration was monitored using carbon dioxide gas analyzer model 200-600 Gow-Mac-Instruments company U.S.A. inside the metal drums.

\section{Calculation of joint action of the plant extract and $\mathrm{CO}_{2}$ :}

For the evaluation of the combined action of the plant extract and $\mathrm{MA}$ of $\mathrm{CO}_{2}$ the following equation adopted by Mansor et al. (1966) was used.

$$
\text { Co-toxicity factor }=\frac{\text { Observed mortality }(\%)-\text { Expected mortality }}{\text { Expected mortality }(\%)} \times 100
$$

This factor was used to classify the results into three categories, positive factor of 20 or more meant potentiation, or synergistic effect, a negative factor of -20 or more meant antagonism and any intermediate value, i.e. between +20 and -20 was considered only an additive effect.

\section{Statistical analysis:}

Dosage mortality response was determined by probit analysis (Finney 1971 using a computer program of Noack and Reichmuth(1978).) 


\section{RESULTS AND DISCUSSION}

Effect of the plant extracts under modified atmosphere (MA) of $20 \pm 5 \% \mathrm{Co}_{2}$ and their combined action with the tested insect species.

The effectiveness of acetone and petroleum ether extracts of certain plants, i.e. Poinciana seeds (Delonix regia), Cinnamon strip bark (Cinnamomum zeylanicum), Cloves flowering buds (Syzygium aromaticum), Mustard seeds (Brassica alba) and Radish seeds (Raphanus sativus), was tested against the adults of Sitophilus oryzae (L.), Rhizopertha dominica (F.) and Tribolium castaneum (Herbst). Tests were carried out in the laboratory at $26 \pm 2^{\circ} \mathrm{C}$ and $60 \pm 5 \%$ R.H.

The various plant extracts were tested alone and under $\mathrm{MA}$ of $20 \pm 5 \% \mathrm{CO}_{2}$ at $0.4 \%$ and $0.8 \%(\mathrm{w} / \mathrm{w})$ concentrations only. Tests were conducted inside fiberglass bins during summer time (grain temp. $=32 \pm 2{ }^{\circ} \mathrm{C}$ ).

The obtained results are shown in Tables (1-20). Results concerning Cotoxicity values results from addition of $20 \pm 5 \% \mathrm{CO}_{2}$ to Poinciana seeds (D. regia) extracts are given in Tables $(1 \& 2)$ results revealed that the $0.4 \& 0.8 \%$ acetone extracts under $20 \pm 5 \% \mathrm{CO}_{2}$ produced additive effect for the three insect species at all exposure periods. Also results revealed that the 0.4 and $0.8 \%(\mathrm{w} / \mathrm{w})$ petroleum ether extracts of Poinciana seeds under $20 \pm 5 \% \mathrm{CO}_{2}$ produced also additive effects for all three insect species at the various exposure periods (Tables $3 \& 4$ ).

Effect of $\mathrm{MA} \mathrm{CO}_{2}$, acetone and petroleum ether extracts of Cinnamom strip bark $C$. zeylanicum alone and in combinations on the percent mortality and their Cotoxicity factors are listed in (Tables 5, 6, 7 and 8) Co-toxicity values presented in these Tables, clearly showed at 1, 2, 3 and 5 days exposure periods additive effects for the two tested concentrations with the three insect species.

Joint action effect of $20 \pm 5 \% \mathrm{CO}_{2}$ plus acetone and petroleum ether extracts of cloves flowering buds ( $S$. aromaticum) to the adults of the three insects infesting stored maize grains inside the fiberglass bins is summarized in Tables (9-12). The results revealed additive effects for the three insect species at all tested exposure periods.

That is to say, carbon dioxide potentiated the toxicity of the extracts against $S$. oryzae, $R$. dominica and $T$. castaneum by using various mixtures of the plant extracts adding $\mathrm{CO}_{2}$ at various exposure periods.

Joint action effect of $20 \pm 5 \% \mathrm{Co}_{2}$ and the extracts of white mustard seeds (Brassica $a l b a)$ for the adults of the three insect species infesting stored maize grains is given in Tables (13-16). 
Results showed additive effects for S. oryzae, R. dominica and T. castaneum after 1, 2, 3 and 5 days exposure periods.

On the other hand, Co-toxicity factors resulting from addition of $\mathrm{CO}_{2}$ to $B$. alba seeds extracts at $(0.4$ and $0.8 \%)$ concentrations of acetone and petroleum ether was greater for $S$. oryzae than in case of $R$. dominica and $T$. castaneum after all exposure periods.

Results concerning co-toxicity factor resulting from adding $20 \pm 5 \% \mathrm{CO}_{2}$ to 0.4 and 0.8 concentrations of the acetone and petroleum ether extract of radish seeds ( $R$. sativus) are listed (Tables 17, 18, 19 and 20). The results indicated additive effects at all exposure time for $S$. oryzae, $R$. dominica and $T$. castaneum adults.

From the above data it could be shown that the joint action of the extracts of the five tested plants under MA of $20 \pm 5 \% \mathrm{Co}_{2}$ produced additive effects for all three insect species at all exposure periods.

Also, the various plant extracts were more toxic against $S$. oryzae adults than other insect species at both concentrations and all exposure periods. Meanwhile, petroleum ether extract was more toxic than the acetone extract. The results obtained for the joint action are in harmony with the findings of other investigators evaluating the combined action of other plant extracts and various modified atmospheres against stored products insects (El-Lakwah et al., 1996; Halawa et al., 1998; Mohamed, 1999; El-Lakwah et al., 2000 and Abd El-Aziz, 2002).

The toxicity effect of these plant extracts is due to the fact that these plants contain L-Azetidi-2-carboxylic acid, Casuarictin, Eugeniln II; Tellimagrandin, Carpacin, Brassinin and S-Methyl-L-Cysteine- S-oxide or substances, which posses antifeedant, repellent or other lead to a moulting disturbance which is often lethal (Cronwell, 1979; Okuda, 1983; Mohandas, 1969; Shimizu, 1986 and Keethaas, 1931). Furthermore, treatment of the various insects with the plant extracts under modified atmosphere of $\mathrm{CO}_{2}$ produced higher mortality values than each treatment alone and proved to be effective against the three insect species. 
Table 1. Joint action effect of $20 \pm 5 \% \mathrm{CO}_{2}$ and $0.4 \%(\mathrm{w} / \mathrm{w})$ acetone extract of Poinciana seeds (Delonix regia) to the adults of some insects infesting stored maize grains inside the fiberglass bins at $32 \pm 2{ }^{\circ} \mathrm{C}$ and $60 \pm 5 \% \mathrm{RH}$.

\begin{tabular}{|c|c|c|c|c|c|c|}
\hline \multirow[b]{2}{*}{ Insect species } & \multirow[b]{2}{*}{$\begin{array}{c}\text { Exposure } \\
\text { period } \\
\text { (days) }\end{array}$} & \multicolumn{3}{|c|}{$\%$ adult mortality } & \multirow[b]{2}{*}{$\begin{array}{c}\text { CO-toxicity } \\
\text { factor }\end{array}$} & \multirow[b]{2}{*}{$\begin{array}{c}\text { Type o } \\
\text { joint } \\
\text { action }\end{array}$} \\
\hline & & $\begin{array}{c}\text { MA of } \\
20 \pm 5 \% \\
\mathrm{CO}_{2} \text { alone }\end{array}$ & $\begin{array}{c}\text { Extract at } \\
0.4 \% \\
(w / w) \\
\text { alone }\end{array}$ & $\begin{array}{c}\text { Extract + } \\
\mathrm{CO}_{2}\end{array}$ & & \\
\hline \multirow{4}{*}{$\begin{array}{c}\text { Sitophilus } \\
\text { oryzae }\end{array}$} & 1 & $6.7 \pm 0.0$ & $42.5 \pm 1.9$ & $51.2 \pm 3.3$ & 4.1 & $d$ \\
\hline & 2 & $33.1 \pm 5.0$ & $83.8 \pm 5.0$ & $100 \pm 0.0$ & 0.0 & $d$ \\
\hline & 3 & $46.7 \pm 6.9$ & $100 \pm 0.0$ & $100 \pm 0.0$ & 0.0 & d \\
\hline & 5 & $52.7 \pm 3.3$ & $100 \pm 0.0$ & $100 \pm 0.0$ & 0.0 & $d$ \\
\hline Control & -- & $0.0 \pm 0.0$ & $0.0 \pm 0.0$ & $0.0 \pm 0.0$ & -- & -- \\
\hline \multirow{4}{*}{$\begin{array}{c}\text { Rhizopertha } \\
\text { dominica }\end{array}$} & 1 & $3.3 \pm 0.0$ & $5.9 \pm 1.9$ & $8.0 \pm 0.0$ & -13.5 & $d$ \\
\hline & 2 & $6.1 \pm 1.9$ & $10.7 \pm 0.0$ & $13.7 \pm 1.9$ & -18.5 & $d$ \\
\hline & 3 & $14.7 \pm 3.3$ & $18.7 \pm 1.9$ & $38.6 \pm 1.9$ & 15.6 & d \\
\hline & 5 & $19.6 \pm 3.0$ & $33.1 \pm 1.9$ & $44 \pm 1.9$ & -16.5 & d \\
\hline Control & -- & $0.0 \pm 0.0$ & $0.0 \pm 0.0$ & $0.0 \pm 0.0$ & -- & -- \\
\hline \multirow{4}{*}{$\begin{array}{l}\text { Tribolium } \\
\text { castaneum }\end{array}$} & 1 & $3.3 \pm 0.0$ & $0.0 \pm 0.0$ & $3.3 \pm 0.0$ & 0.0 & d \\
\hline & 2 & $7.2 \pm 0.0$ & $10 \pm 1.9$ & $14 \pm 3.3$ & -18.6 & d \\
\hline & 3 & $16.8 \pm 1.9$ & $19.9 \pm 2.0$ & $33.2 \pm 1.9$ & -9.5 & $d$ \\
\hline & 5 & $27.6 \pm 3.3$ & $29.3 \pm 3.3$ & $50 \pm 1.9$ & -12.1 & $d$ \\
\hline Control & -- & $0.0 \pm 0.0$ & $0.0 \pm 0.0$ & $0.0 \pm 0.0$ & -- & -- \\
\hline
\end{tabular}

$\mathrm{d}=$ additive effect. 
Table 2. Joint action effect of $20 \pm 5 \% \mathrm{CO}_{2}$ and $0.8 \%(\mathrm{w} / \mathrm{w})$ acetone extract of Poinciana seeds (Delonix regia) to the adults of some insects infesting stored maize grains inside the fiberglass bins at $32 \pm 2^{\circ} \mathrm{C}$ and $60 \pm$ $5 \% \mathrm{RH}$.

\begin{tabular}{|c|c|c|c|c|c|c|}
\hline \multirow[b]{2}{*}{ Insect species } & \multirow{2}{*}{$\begin{array}{l}\text { Exposure } \\
\text { period } \\
\text { (days) }\end{array}$} & \multicolumn{3}{|c|}{$\%$ adult mortality } & \multirow{2}{*}{$\begin{array}{l}\text { CO- } \\
\text { toxicity } \\
\text { factor }\end{array}$} & \multirow{2}{*}{$\begin{array}{c}\text { Type of } \\
\text { joint } \\
\text { action }\end{array}$} \\
\hline & & $\begin{array}{c}20 \pm 5 \% \mathrm{CO}_{2} \\
\text { alone } \\
\end{array}$ & $\begin{array}{c}0.8 \%(\mathrm{w} / \mathrm{w}) \\
\text { alone } \\
\end{array}$ & $\begin{array}{c}\text { Extract }+ \\
\mathrm{CO}_{2}\end{array}$ & & \\
\hline \multirow{4}{*}{$\begin{array}{c}\text { Sitophilus } \\
\text { oryzae }\end{array}$} & 1 & $6.7 \pm 0.0$ & $57.4 \pm 1.9$ & $70 \pm 3.3$ & 9.2 & $d$ \\
\hline & 2 & $33.1 \pm 5.0$ & $100 \pm 0.0$ & $100 \pm 0.0$ & 0.0 & d \\
\hline & 3 & $46.7 \pm 6.9$ & $100 \pm 0.0$ & $100 \pm 0.0$ & 0.0 & $d$ \\
\hline & 5 & $52.7 \pm 3.3$ & $100 \pm 0.0$ & $100 \pm 0.0$ & 0.0 & d \\
\hline Control & -- & $0.0 \pm 0.0$ & $0.0 \pm 0.0$ & $0.0 \pm 0.0$ & -- & -- \\
\hline \multirow{4}{*}{$\begin{array}{c}\text { Rhizopertha } \\
\text { dominica }\end{array}$} & 1 & $3.3 \pm 0.0$ & $60 \pm 1.9$ & $67.8 \pm 3.3$ & 7.1 & d \\
\hline & 2 & $6.1 \pm 1.9$ & $68.2 \pm 1.9$ & $73.7 \pm 1.9$ & -8.0 & d \\
\hline & 3 & $14.7 \pm 3.3$ & $75 \pm 1.9$ & $78.5 \pm 3.3$ & -12.5 & d \\
\hline & 5 & $19.6 \pm 3.0$ & $77.3 \pm 1.9$ & $83.2 \pm 1.9$ & -14.1 & $d$ \\
\hline Control & -- & $0.0 \pm 0.0$ & $0.0 \pm 0.0$ & $0.0 \pm 0.0$ & -- & -- \\
\hline \multirow{4}{*}{$\begin{array}{l}\text { Tribolium } \\
\text { castaneum }\end{array}$} & 1 & $3.3 \pm 0.0$ & $3.3 \pm 0.0$ & $5.7 \pm 0.0$ & -13.6 & d \\
\hline & 2 & $7.2 \pm 0.0$ & $11.7 \pm 1.9$ & $22.2 \pm 1.9$ & 17.5 & d \\
\hline & 3 & $16.8 \pm 1.9$ & $24.4 \pm 2.0$ & $39.6 \pm 3.3$ & -3.9 & d \\
\hline & 5 & $27.6 \pm 3.3$ & $36.4 \pm 3.3$ & $54 \pm 3.3$ & -15.6 & d \\
\hline Control & -- & $0.0 \pm 0.0$ & $0.0 \pm 0.0$ & $0.0 \pm 0.0$ & -- & -- \\
\hline
\end{tabular}

$\mathrm{d}=$ additive effect. 
Table 3. Joint action effect of $20 \pm 5 \% \mathrm{CO}_{2}$ and $0.4 \%(\mathrm{w} / \mathrm{w})$ petroleum ether extract of Poinciana seeds (Delonix regia) to the adults of some insects infesting stored maize grains inside the fiberglass bins at $32 \pm 2{ }^{\circ} \mathrm{C}$ and $60 \pm 5 \% \mathrm{RH}$.

\begin{tabular}{|c|c|c|c|c|c|c|}
\hline \multirow[b]{2}{*}{ Insect species } & \multirow{2}{*}{$\begin{array}{c}\text { Exposure } \\
\text { period } \\
\text { (days) }\end{array}$} & \multicolumn{3}{|c|}{$\%$ adult mortality } & \multirow{2}{*}{$\begin{array}{l}\text { CO- } \\
\text { toxicity } \\
\text { factor }\end{array}$} & \multirow{2}{*}{$\begin{array}{c}\text { Type of } \\
\text { joint } \\
\text { action }\end{array}$} \\
\hline & & $\begin{array}{c}\text { MA of } \\
20 \pm 5 \% \mathrm{CO}_{2} \\
\text { alone }\end{array}$ & $\begin{array}{c}\text { Extract at } \\
0.4 \%(\mathrm{w} / \mathrm{w}) \\
\text { alone }\end{array}$ & $\begin{array}{c}\text { Extract }+ \\
\qquad \mathrm{CO}_{2}\end{array}$ & & \\
\hline \multirow{4}{*}{$\begin{array}{c}\text { Sitophilus } \\
\text { oryzae }\end{array}$} & 1 & $6.7 \pm 0.0$ & $35.1 \pm 1.9$ & $66.7 \pm 3.3$ & 11.5 & $d$ \\
\hline & 2 & $33.1 \pm 5.0$ & $90 \pm 1.9$ & $100 \pm 0.0$ & 0.0 & $d$ \\
\hline & 3 & $46.7 \pm 6.9$ & $100 \pm 0.0$ & $100 \pm 0.0$ & 0.0 & d \\
\hline & 5 & $52.7 \pm 3.3$ & $100 \pm 0.0$ & $100 \pm 0.0$ & 0.0 & $d$ \\
\hline Control & -- & $0.0 \pm 0.0$ & $0.0 \pm 0.0$ & $0.0 \pm 0.0$ & -- & -- \\
\hline \multirow{4}{*}{$\begin{array}{c}\text { Rhizopertha } \\
\text { dominica }\end{array}$} & 1 & $3.3 \pm 0.0$ & $3.3 \pm 0.0$ & $6.7 \pm 0.0$ & 1.5 & $d$ \\
\hline & 2 & $6.1 \pm 1.9$ & $13.8 \pm 1.9$ & $16.8 \pm 1.9$ & -15.6 & $d$ \\
\hline & 3 & $14 \pm 3.3$ & $25.3 \pm 1.9$ & $32.3 \pm 1.9$ & -17.8 & $d$ \\
\hline & 5 & $19.6 \pm 3.0$ & $58.6 \pm 1.9$ & $66.2 \pm 1.9$ & -15.3 & $d$ \\
\hline Control & -- & $0.0 \pm 0.0$ & $0.0 \pm 0.0$ & $0.0 \pm 0.0$ & -- & -- \\
\hline \multirow{4}{*}{$\begin{array}{l}\text { Tribolium } \\
\text { castaneum }\end{array}$} & 1 & $3.3 \pm 0.0$ & $3.3 \pm 0.0$ & $6.7 \pm 0.0$ & 1.5 & $d$ \\
\hline & 2 & $7.2 \pm 0.0$ & $10.7 \pm 1.9$ & $15.5 \pm 1.9$ & -13.4 & $d$ \\
\hline & 3 & $16.8 \pm 1.9$ & $17.7 \pm 1.9$ & $28.3 \pm 3.3$ & -18.0 & d \\
\hline & 5 & $27.6 \pm 3.3$ & $29.6 \pm 3.3$ & $45.8 \pm 3.3$ & -19.9 & $d$ \\
\hline Control & -- & $0.0 \pm 0.0$ & $0.0 \pm 0.0$ & $0.0 \pm 0.0$ & -- & -- \\
\hline
\end{tabular}

$\mathrm{d}=$ additive effect. 
Table 4. Joint action effect of $20 \pm 5 \% \mathrm{CO}_{2}$ and $0.8 \%(\mathrm{w} / \mathrm{w})$ petroleum ether extract of Poinciana seeds (Delonix regia) to the adults of some insects infesting stored maize grains inside the fiberglass bins at $32 \pm 2{ }^{\circ} \mathrm{C}$ and $60 \pm 5 \% \mathrm{RH}$.

\begin{tabular}{|c|c|c|c|c|c|c|}
\hline \multirow[b]{2}{*}{ Insect species } & \multirow{2}{*}{$\begin{array}{c}\text { Exposure } \\
\text { period } \\
\text { (days) }\end{array}$} & \multicolumn{3}{|c|}{$\%$ adult mortality } & \multirow{2}{*}{$\begin{array}{l}\text { CO- } \\
\text { toxicity } \\
\text { factor }\end{array}$} & \multirow{2}{*}{$\begin{array}{c}\text { Type of } \\
\text { joint } \\
\text { action }\end{array}$} \\
\hline & & $\begin{array}{c}\text { MA of } \\
20 \pm 5 \% \mathrm{CO}_{2} \\
\text { alone }\end{array}$ & $\begin{array}{c}\text { Extract at } \\
0.8 \%(\mathrm{w} / \mathrm{w}) \\
\text { alone }\end{array}$ & Extract $+\mathrm{CO}_{2}$ & & \\
\hline \multirow{4}{*}{$\begin{array}{c}\text { Sitophilus } \\
\text { oryzae }\end{array}$} & 1 & $6.7 \pm 0.0$ & $71.5 \pm 1.9$ & $86.7 \pm 8.4$ & 10.9 & d \\
\hline & 2 & $33.1 \pm 5.0$ & $100 \pm 0.0$ & $100 \pm 0.0$ & 0.0 & $d$ \\
\hline & 3 & $46.7 \pm 6.9$ & $100 \pm 0.0$ & $100 \pm 0.0$ & 0.0 & $d$ \\
\hline & 5 & $52.7 \pm 3.3$ & $100 \pm 0.0$ & $100 \pm 0.0$ & 0.0 & $d$ \\
\hline Control & -- & $0.0 \pm 0.0$ & $0.0 \pm 0.0$ & $0.0 \pm 0.0$ & -- & -- \\
\hline \multirow{4}{*}{$\begin{array}{c}\text { Rhizopertha } \\
\text { dominica }\end{array}$} & 1 & $3.3 \pm 0.0$ & $58.6 \pm 3.3$ & $66.7 \pm 3.3$ & 7.8 & $d$ \\
\hline & 2 & $6.1 \pm 1.9$ & $36.1 \pm 1.9$ & $71.5 \pm 1.9$ & 3.3 & $d$ \\
\hline & 3 & $14 \pm 3.3$ & $70.1 \pm 1.9$ & $79.0 \pm 1.9$ & -6.1 & d \\
\hline & 5 & $19.6 \pm 3.0$ & $78.1 \pm 1.9$ & $92.5 \pm 1.9$ & -5.3 & $d$ \\
\hline Control & -- & $0.0 \pm 0.0$ & $0.0 \pm 0.0$ & $0.0 \pm 0.0$ & -- & -- \\
\hline \multirow{4}{*}{$\begin{array}{l}\text { Tribolium } \\
\text { castaneum }\end{array}$} & 1 & $3.3 \pm 0.0$ & $6.7 \pm 0.0$ & $9.4 \pm 1.9$ & -6.0 & $d$ \\
\hline & 2 & $7.2 \pm 0.0$ & $13.3 \pm 1.9$ & $18.8 \pm 1.9$ & -8.3 & d \\
\hline & 3 & $16.8 \pm 1.9$ & $20.1 \pm 1.9$ & $30.7 \pm 3.8$ & -16.8 & $d$ \\
\hline & 5 & $27.6 \pm 3.3$ & $39.2 \pm 3.3$ & $53.7 \pm 3.3$ & -19.6 & d \\
\hline Control & -- & $0.0 \pm 0.0$ & $0.0 \pm 0.0$ & $0.0 \pm 0.0$ & -- & -- \\
\hline
\end{tabular}

$\mathrm{d}=$ additive effect. 
Table 5. Joint action effect of $20 \pm 5 \% \mathrm{CO}_{2}$ and $0.4 \%(\mathrm{w} / \mathrm{w})$ acetone extract of Cinnamom strip bark (Cinnamomum zeylanicum) to the adults of some insects infesting stored maize grains inside the fiberglass bins at $32 \pm 2^{\circ} \mathrm{C}$ and $60 \pm 5 \% \mathrm{RH}$.

\begin{tabular}{|c|c|c|c|c|c|c|}
\hline \multirow[b]{2}{*}{ Insect species } & \multirow[b]{2}{*}{$\begin{array}{c}\text { Exposure } \\
\text { period } \\
\text { (days) }\end{array}$} & \multicolumn{3}{|c|}{$\%$ adult mortality } & \multirow[b]{2}{*}{$\begin{array}{l}\text { CO- } \\
\text { toxicity } \\
\text { factor }\end{array}$} & \multirow[b]{2}{*}{$\begin{array}{c}\text { Type of } \\
\text { joint } \\
\text { action }\end{array}$} \\
\hline & & $\begin{array}{c}\mathrm{MA} \text { of } 20 \pm 5 \% \\
\mathrm{CO}_{2} \text { alone }\end{array}$ & $\begin{array}{c}\text { Extract at } \\
0.4 \%(\mathrm{w} / \mathrm{w}) \\
\text { alone }\end{array}$ & $\begin{array}{c}\text { Extract }+ \\
\mathrm{CO}_{2}\end{array}$ & & \\
\hline \multirow{4}{*}{$\begin{array}{c}\text { Sitophilus } \\
\text { oryzae }\end{array}$} & 1 & $6.7 \pm 0.0$ & $16.7 \pm 3.3$ & $28.0 \pm 1.9$ & 19.6 & d \\
\hline & 2 & $33.1 \pm 5.0$ & $52.5 \pm 1.9$ & $100 \pm 0.0$ & 16.8 & d \\
\hline & 3 & $46.7 \pm 6.9$ & $89.9 \pm 3.8$ & $100 \pm 0.0$ & 0.0 & $d$ \\
\hline & 5 & $52.7 \pm 3.3$ & $95.0 \pm 1.9$ & $100 \pm 0.0$ & 0.0 & d \\
\hline Control & -- & $0.0 \pm 0.0$ & $0.0 \pm 0.0$ & $0.0 \pm 0.0$ & -- & -- \\
\hline \multirow{4}{*}{$\begin{array}{c}\text { Rhizopertha } \\
\text { dominica }\end{array}$} & 1 & $3.3 \pm 0.0$ & $3.6 \pm 0.0$ & $6.7 \pm 0.0$ & -2.9 & d \\
\hline & 2 & $6.1 \pm 1.9$ & $10.7 \pm 1.9$ & $14.0 \pm 3.3$ & -16.7 & d \\
\hline & 3 & $14.0 \pm 3.3$ & $20.1 \pm 1.9$ & $27.2 \pm 1.9$ & -20.0 & d \\
\hline & 5 & $19.6 \pm 3.0$ & $32.1 \pm 1.9$ & $41.7 \pm 1.9$ & -19.3 & d \\
\hline Control & -- & $0.0 \pm 0.0$ & $0.0 \pm 0.0$ & $0.0 \pm 0.0$ & -- & -- \\
\hline \multirow{4}{*}{$\begin{array}{l}\text { Tribolium } \\
\text { castaneum }\end{array}$} & 1 & $3.3 \pm 0.0$ & $0.0 \pm 0.0$ & $3.9 \pm 0.0$ & 9.1 & d \\
\hline & 2 & $7.2 \pm 0.0$ & $8.3 \pm 1.9$ & $14 \pm 1.9$ & -9.7 & d \\
\hline & 3 & $16.8 \pm 1.9$ & $18.0 \pm 1.9$ & $28 \pm 1.9$ & -19.5 & d \\
\hline & 5 & $27.6 \pm 3.3$ & $31.1 \pm 1.9$ & $47 \pm 1.9$ & -20 & $d$ \\
\hline Control & -- & $0.0 \pm 0.0$ & $0.0 \pm 0.0$ & $0.0 \pm 0.0$ & -- & -- \\
\hline
\end{tabular}

$\mathrm{d}=$ additive effect. 
Table 6. Joint action effect of $20 \pm 5 \% \mathrm{CO}_{2}$ and $0.8 \%(\mathrm{w} / \mathrm{w})$ acetone extract of Cinnamom strip bark (Cinnamomum zeylanicum) to the adults of some insects infesting stored maize grains inside the fiberglass bins at $32 \pm 2^{\circ} \mathrm{C}$ and $60 \pm 5 \% \mathrm{RH}$.

\begin{tabular}{|c|c|c|c|c|c|c|}
\hline \multirow[b]{2}{*}{ Insect species } & \multirow[b]{2}{*}{$\begin{array}{c}\text { Exposure } \\
\text { period } \\
\text { (days) }\end{array}$} & \multicolumn{3}{|c|}{$\%$ adult mortality } & \multirow[b]{2}{*}{$\begin{array}{l}\text { CO- } \\
\text { toxicity } \\
\text { factor }\end{array}$} & \multirow[b]{2}{*}{$\begin{array}{c}\text { Type of } \\
\text { joint } \\
\text { action }\end{array}$} \\
\hline & & $\begin{array}{c}\mathrm{MA} \text { of } \\
20 \pm 5 \% \mathrm{CO}_{2} \\
\text { alone }\end{array}$ & $\begin{array}{c}\text { Extract at } \\
0.8 \% \\
(\mathrm{w} / \mathrm{w}) \\
\text { alone }\end{array}$ & $\begin{array}{c}\text { Extract }+ \\
\qquad \mathrm{CO}_{2}\end{array}$ & & \\
\hline \multirow{4}{*}{$\begin{array}{c}\text { Sitophilus } \\
\text { oryzae }\end{array}$} & 1 & $6.7 \pm 0.0$ & $37.4 \pm 1.9$ & $52.9 \pm 1.9$ & 19.9 & $\mathrm{~d}$ \\
\hline & 2 & $33.1 \pm 5.0$ & $76.2 \pm 1.9$ & $100 \pm 0.0$ & 0.0 & $d$ \\
\hline & 3 & $46.7 \pm 6.9$ & $95 \pm 5.0$ & $100 \pm 0.0$ & 0.0 & $d$ \\
\hline & 5 & $52.7 \pm 3.3$ & $96.2 \pm 0.0$ & $100 \pm 0.0$ & 0.0 & d \\
\hline Control & -- & $0.0 \pm 0.0$ & $0.0 \pm 0.0$ & $0.0 \pm 0.0$ & -- & -- \\
\hline \multirow{4}{*}{$\begin{array}{c}\text { Rhizopertha } \\
\text { dominica }\end{array}$} & 1 & $3.3 \pm 0.0$ & $5.6 \pm 0.0$ & $8.9 \pm 0.0$ & 0.0 & $\mathrm{~d}$ \\
\hline & 2 & $6.1 \pm 1.9$ & $13.6 \pm 1.9$ & $18.5 \pm 1.9$ & -6.0 & $d$ \\
\hline & 3 & $14.0 \pm 3.3$ & $23.7 \pm 1.9$ & $31.0 \pm 3.3$ & -17.8 & $d$ \\
\hline & 5 & $19.6 \pm 3.0$ & $35.6 \pm 1.9$ & $50.0 \pm 3.3$ & -9.4 & $\mathrm{~d}$ \\
\hline Control & -- & $0.0 \pm 0.0$ & $0.0 \pm 0.0$ & $0.0 \pm 0.0$ & -- & -- \\
\hline \multirow{4}{*}{$\begin{array}{l}\text { Tribolium } \\
\text { castaneum }\end{array}$} & 1 & $3.3 \pm 0.0$ & $5.5 \pm 0.0$ & $8.4 \pm 1.9$ & -4.5 & $d$ \\
\hline & 2 & $7.2 \pm 0.0$ & $10 \pm 1.9$ & $16.8 \pm 0.0$ & -2.3 & $\mathrm{~d}$ \\
\hline & 3 & $16.8 \pm 1.9$ & $20.4 \pm 3.3$ & $30 \pm 1.9$ & -19.3 & d \\
\hline & 5 & $27.6 \pm 3.3$ & $36.6 \pm 1.9$ & $77 \pm 5.6$ & 19.9 & d \\
\hline Control & -- & $0.0 \pm 0.0$ & $0.0 \pm 0.0$ & $0.0 \pm 0.0$ & -- & -- \\
\hline
\end{tabular}

$\mathrm{d}=$ additive effect. 
Table 7. Joint action effect of $20 \pm 5 \% \mathrm{CO}_{2}$ and $0.4 \%(\mathrm{w} / \mathrm{w})$ petroleum ether extract of Cinnamom strip bark (Cinnamomum zeylanicum) to the adults of some insects infesting stored maize grains inside the fiberglass bins at $32 \pm 2^{\circ} \mathrm{C}$ and $60 \pm 5 \% \mathrm{RH}$.

\begin{tabular}{|c|c|c|c|c|c|c|}
\hline \multirow[b]{2}{*}{ Insect species } & \multirow{2}{*}{$\begin{array}{l}\text { Exposure } \\
\text { period } \\
\text { (days) }\end{array}$} & \multicolumn{3}{|c|}{$\%$ adult mortality } & \multirow{2}{*}{$\begin{array}{l}\text { CO- } \\
\text { toxicity } \\
\text { factor }\end{array}$} & \multirow{2}{*}{$\begin{array}{c}\text { Type of } \\
\text { joint } \\
\text { action }\end{array}$} \\
\hline & & $\begin{array}{c}\mathrm{MA} \text { of } 20 \pm 5 \% \\
\mathrm{CO}_{2} \text { alone }\end{array}$ & $\begin{array}{c}0.4 \%(\mathrm{w} / \mathrm{w}) \\
\text { alone }\end{array}$ & $\begin{array}{c}\text { Extract + } \\
\mathrm{CO}_{2}\end{array}$ & & \\
\hline \multirow{4}{*}{$\begin{array}{c}\text { Sitophilus } \\
\text { oryzae }\end{array}$} & 1 & $6.7 \pm 0.0$ & $51.2 \pm 3.3$ & $28.0 \pm 1.9$ & -6.9 & d \\
\hline & 2 & $33.1 \pm 5.0$ & $88.7 \pm 3.3$ & $100 \pm 0.0$ & 0.0 & $\mathrm{~d}$ \\
\hline & 3 & $46.7 \pm 6.9$ & $91.2 \pm 1.9$ & $100 \pm 0.0$ & 0.0 & d \\
\hline & 5 & $52.7 \pm 3.3$ & $100 \pm 0.0$ & $100 \pm 0.0$ & 0.0 & d \\
\hline Control & -- & $0.0 \pm 0.0$ & $0.0 \pm 0.0$ & $0.0 \pm 0.0$ & -- & -- \\
\hline \multirow{4}{*}{$\begin{array}{c}\text { Rhizopertha } \\
\text { dominica }\end{array}$} & 1 & $3.3 \pm 0.0$ & $64.2 \pm 1.9$ & $75.0 \pm 1.9$ & 11.1 & d \\
\hline & 2 & $6.1 \pm 1.9$ & $65.4 \pm 1.9$ & $76.2 \pm 1.9$ & 6.6 & d \\
\hline & 3 & $14.0 \pm 3.3$ & $71.5 \pm 1.9$ & $79.9 \pm 1.9$ & -6.5 & d \\
\hline & 5 & $19.6 \pm 3.0$ & $75.3 \pm 1.9$ & $79.9 \pm 1.9$ & -15.8 & d \\
\hline Control & -- & $0.0 \pm 0.0$ & $0.0 \pm 0.0$ & $0.0 \pm 0.0$ & -- & -- \\
\hline \multirow{4}{*}{$\begin{array}{l}\text { Tribolium } \\
\text { castaneum }\end{array}$} & 1 & $3.3 \pm 0.0$ & $0.0 \pm 0.0$ & $3.9 \pm 3.3$ & 18.1 & d \\
\hline & 2 & $7.2 \pm 0.0$ & $14 \pm 1.9$ & $23.3 \pm 1.9$ & 9.9 & d \\
\hline & 3 & $16.8 \pm 1.9$ & $24.6 \pm 1.9$ & $33 \pm 1.9$ & -20.0 & $\mathrm{~d}$ \\
\hline & 5 & $27.6 \pm 3.3$ & $42.2 \pm 5.0$ & $61 \pm 3.3$ & -12.6 & $\mathrm{~d}$ \\
\hline Control & -- & $0.0 \pm 0.0$ & $0.0 \pm 0.0$ & $0.0 \pm 0.0$ & -- & -- \\
\hline
\end{tabular}

$\mathrm{d}=$ additive effect. 
Table 8. Joint action effect of $20 \pm 5 \% \mathrm{CO}_{2}$ and $0.8 \%(\mathrm{w} / \mathrm{w})$ petroleum ether extract of Cinnamom strip bark (Cinnamomum zeylanicum) to the adults of some insects infesting stored maize grains inside the fiberglass bins at $32 \pm 2^{\circ} \mathrm{C}$ and $60 \pm 5 \% \mathrm{RH}$.

\begin{tabular}{|c|c|c|c|c|c|c|}
\hline \multirow[b]{2}{*}{ Insect species } & \multirow{2}{*}{$\begin{array}{c}\text { Exposure } \\
\text { period } \\
\text { (days) }\end{array}$} & \multicolumn{3}{|c|}{$\%$ adult mortality } & \multirow{2}{*}{$\begin{array}{l}\text { CO- } \\
\text { toxicity } \\
\text { factor }\end{array}$} & \multirow{2}{*}{$\begin{array}{c}\text { Type of } \\
\text { joint } \\
\text { action }\end{array}$} \\
\hline & & $\begin{array}{c}\mathrm{MA} \text { of } 20 \pm 5 \% \\
\mathrm{CO}_{2} \text { alone }\end{array}$ & $\begin{array}{c}0.8 \%(\mathrm{w} / \mathrm{w}) \\
\text { alone }\end{array}$ & $\begin{array}{c}\text { Extract }+ \\
\mathrm{CO}_{2}\end{array}$ & & \\
\hline \multirow{4}{*}{$\begin{array}{c}\text { Sitophilus } \\
\text { oryzae }\end{array}$} & 1 & $6.7 \pm 0.0$ & $71.3 \pm 3.8$ & $77.5 \pm 3.3$ & -0.6 & d \\
\hline & 2 & $33.1 \pm 5.0$ & $91.2 \pm 1.9$ & $100 \pm 0.0$ & 0.0 & d \\
\hline & 3 & $46.7 \pm 6.9$ & $98.7 \pm 1.9$ & $100 \pm 0.0$ & 0.0 & d \\
\hline & 5 & $52.7 \pm 3.3$ & $100 \pm 0.0$ & $100 \pm 0.0$ & 0.0 & d \\
\hline Control & -- & $0.0 \pm 0.0$ & $0.0 \pm 0.0$ & $0.0 \pm 0.0$ & -- & -- \\
\hline \multirow{4}{*}{$\begin{array}{c}\text { Rhizopertha } \\
\text { dominica }\end{array}$} & 1 & $3.3 \pm 0.0$ & $75.3 \pm 1.9$ & $86.2 \pm 1.9$ & 9.4 & d \\
\hline & 2 & $6.1 \pm 1.9$ & $77.7 \pm 3.3$ & $91.2 \pm 3.3$ & 8.8 & d \\
\hline & 3 & $14.0 \pm 3.3$ & $70.1 \pm 1.9$ & $91.2 \pm 3.3$ & -1.9 & d \\
\hline & 5 & $19.6 \pm 3.0$ & $82 \pm 1.9$ & $91.2 \pm 3.3$ & -8.8 & d \\
\hline Control & -- & $0.0 \pm 0.0$ & $0.0 \pm 0.0$ & $0.0 \pm 0.0$ & -- & -- \\
\hline \multirow{4}{*}{$\begin{array}{l}\text { Tribolium } \\
\text { castaneum }\end{array}$} & 1 & $3.3 \pm 0.0$ & $6.6 \pm 0.0$ & $11.6 \pm 1.9$ & 17.1 & d \\
\hline & 2 & $7.2 \pm 0.0$ & $16.7 \pm 0.0$ & $24.6 \pm 1.9$ & 2.9 & d \\
\hline & 3 & $16.8 \pm 1.9$ & $25.8 \pm 3.3$ & $36.3 \pm 3.3$ & -14.8 & d \\
\hline & 5 & $27.6 \pm 3.3$ & $61.1 \pm 1.9$ & $78.6 \pm 6.7$ & -11.4 & d \\
\hline Control & -- & $0.0 \pm 0.0$ & $0.0 \pm 0.0$ & $0.0 \pm 0.0$ & -- & -- \\
\hline
\end{tabular}

$\mathrm{d}=$ additive effect. 
Table 9. Joint action effect of $20 \pm 5 \% \mathrm{CO}_{2}$ and $0.4 \%(\mathrm{w} / \mathrm{w})$ acetone extract of Cloves flowering bud (Syzygium aromaticum) to the adults of some insects infesting stored maize grains inside the fiberglass bins at $32 \pm 2{ }^{\circ} \mathrm{C}$ and 60 $\pm 5 \% \mathrm{RH}$.

\begin{tabular}{|c|c|c|c|c|c|c|}
\hline \multirow[b]{2}{*}{ Insect species } & \multirow[b]{2}{*}{$\begin{array}{c}\text { Exposure } \\
\text { period (days) }\end{array}$} & \multicolumn{3}{|c|}{$\%$ adult mortality } & \multirow[b]{2}{*}{$\begin{array}{l}\text { CO- } \\
\text { toxicity } \\
\text { factor }\end{array}$} & \multirow[b]{2}{*}{$\begin{array}{c}\text { Type of } \\
\text { joint } \\
\text { action }\end{array}$} \\
\hline & & $\begin{array}{c}\text { MA of } \\
20 \pm 5 \% \\
\text { CO2 alone }\end{array}$ & $\begin{array}{l}0.4 \% \\
(w / w) \\
\text { alone }\end{array}$ & $\begin{array}{c}\text { Extract }+ \\
\qquad \mathrm{CO} 2\end{array}$ & & \\
\hline \multirow{4}{*}{$\begin{array}{l}\text { Sitophilus } \\
\text { oryzae }\end{array}$} & 1 & $6.7 \pm 0.0$ & $53.1 \pm 1.9$ & $61.7 \pm 1.9$ & 3.2 & d \\
\hline & 2 & $33.1 \pm 5.0$ & $87.6 \pm 1.9$ & $100 \pm 0.0$ & 0.0 & d \\
\hline & 3 & $46.7 \pm 6.9$ & $100 \pm 0.0$ & $100 \pm 0.0$ & 0.0 & $d$ \\
\hline & 5 & $52.7 \pm 3.3$ & $100 \pm 0.0$ & $100 \pm 0.0$ & 0.0 & $d$ \\
\hline Control & -- & $0.0 \pm 0.0$ & $0.0 \pm 0.0$ & $0.0 \pm 0.0$ & -- & -- \\
\hline \multirow{4}{*}{$\begin{array}{l}\text { Rhizopertha } \\
\text { dominica }\end{array}$} & 1 & $3.3 \pm 0.0$ & $14.0 \pm 1.9$ & $16.7 \pm 3.3$ & -3.5 & $d$ \\
\hline & 2 & $6.1 \pm 1.9$ & $65.8 \pm 1.9$ & $74.7 \pm 1.9$ & 3.9 & d \\
\hline & 3 & $14.0 \pm 3.3$ & $76.4 \pm 2.0$ & $89.8 \pm 2.0$ & -0.7 & $d$ \\
\hline & 5 & $19.6 \pm 3.0$ & $84.6 \pm 1.9$ & $96.7 \pm 1.9$ & -3.3 & d \\
\hline Control & -- & $0.0 \pm 0.0$ & $0.0 \pm 0.0$ & $0.0 \pm 0.0$ & -- & -- \\
\hline \multirow{4}{*}{$\begin{array}{l}\text { Tribolium } \\
\text { castaneum }\end{array}$} & 1 & $3.3 \pm 0.0$ & $0.0 \pm 0.0$ & $3.6 \pm 0.0$ & 9.1 & d \\
\hline & 2 & $7.2 \pm 0.0$ & $13.0 \pm 1.9$ & $24.0 \pm 1.9$ & 18.8 & $d$ \\
\hline & 3 & $16.8 \pm 1.9$ & $21.4 \pm 3.3$ & $33.3 \pm 1.9$ & -12.8 & $d$ \\
\hline & 5 & $27.6 \pm 3.3$ & $60.6 \pm 3.3$ & $71.0 \pm 3.3$ & -19.5 & d \\
\hline Control & -- & $0.0 \pm 0.0$ & $0.0 \pm 0.0$ & $0.0 \pm 0.0$ & -- & -- \\
\hline
\end{tabular}

$\mathrm{d}=$ additive effect. 
Table 10. Joint action effect of $20 \pm 5 \% \mathrm{CO}_{2}$ and $0.8 \%(\mathrm{w} / \mathrm{w})$ acetone extract of Cloves flowering bud (Syzygium aromaticum) to the adults of some insects infesting stored maize grains inside the fiberglass bins at $32 \pm 2^{\circ} \mathrm{C}$ and $60 \pm 5 \% \mathrm{RH}$.

\begin{tabular}{|c|c|c|c|c|c|c|}
\hline \multirow[b]{2}{*}{ Insect species } & \multirow[b]{2}{*}{$\begin{array}{l}\text { Exposure } \\
\text { period } \\
\text { (days) }\end{array}$} & \multicolumn{3}{|c|}{$\%$ adult mortality } & \multirow[b]{2}{*}{$\begin{array}{l}\text { CO- } \\
\text { toxicity } \\
\text { factor }\end{array}$} & \multirow[b]{2}{*}{$\begin{array}{c}\text { Type of } \\
\text { joint } \\
\text { action }\end{array}$} \\
\hline & & $\begin{array}{c}\mathrm{MA} \text { of } \\
20 \pm 5 \% \mathrm{CO}_{2} \\
\text { alone }\end{array}$ & $\begin{array}{c}\text { Extract at } \\
0.8 \% \\
(\mathrm{w} / \mathrm{w}) \\
\text { alone }\end{array}$ & $\begin{array}{c}\text { Extract + } \\
\mathrm{CO}_{2}\end{array}$ & & \\
\hline \multirow{4}{*}{ Sitophilus } & 1 & $6.7 \pm 0.0$ & $62.8 \pm 1.9$ & $67.8 \pm 5.0$ & -2.4 & $d$ \\
\hline & 2 & $33.1 \pm 5.0$ & $100 \pm 0.0$ & $100 \pm 0.0$ & 0.0 & $d$ \\
\hline & 3 & $46.7 \pm 6.9$ & $100 \pm 0.0$ & $100 \pm 0.0$ & 0.0 & d \\
\hline & 5 & $52.7 \pm 3.3$ & $100 \pm 0.0$ & $100 \pm 0.0$ & 0.0 & $d$ \\
\hline Control & -- & $0.0 \pm 0.0$ & $0.0 \pm 0.0$ & $0.0 \pm 0.0$ & -- & -- \\
\hline \multirow{4}{*}{$\begin{array}{c}\text { Rhizopertha } \\
\text { dominica }\end{array}$} & 1 & $3.3 \pm 0.0$ & $20.0 \pm 1.9$ & $21.5 \pm 1.9$ & -7.7 & d \\
\hline & 2 & $6.1 \pm 1.9$ & $69.3 \pm 1.9$ & $78.4 \pm 1.9$ & 4.0 & $d$ \\
\hline & 3 & $14.0 \pm 3.3$ & $82.3 \pm 3.3$ & $93.3 \pm 3.3$ & -3.1 & $d$ \\
\hline & 5 & $19.6 \pm 3.0$ & $88.2 \pm 1.9$ & $98.6 \pm 2.0$ & -1.4 & d \\
\hline Control & -- & $0.0 \pm 0.0$ & $0.0 \pm 0.0$ & $0.0 \pm 0.0$ & -- & -- \\
\hline \multirow{4}{*}{$\begin{array}{l}\text { Tribolium } \\
\text { castaneum }\end{array}$} & 1 & $3.3 \pm 0.0$ & $5.0 \pm 0.0$ & $9.8 \pm 1.9$ & 18.0 & d \\
\hline & 2 & $7.2 \pm 0.0$ & $22.8 \pm 1.9$ & $36.0 \pm 5.0$ & 20 & d \\
\hline & 3 & $16.8 \pm 1.9$ & $57.1 \pm 1.9$ & $64.3 \pm 5.0$ & -13.0 & d \\
\hline & 5 & $27.6 \pm 3.3$ & $78.5 \pm 6.7$ & $89.2 \pm 3.3$ & -10.8 & $d$ \\
\hline Control & -- & $0.0 \pm 0.0$ & $0.0 \pm 0.0$ & $0.0 \pm 0.0$ & -- & -- \\
\hline
\end{tabular}

$\mathrm{d}$ = additive effect. 
Table 11. Joint action effect of $20 \pm 5 \% \mathrm{CO}_{2}$ and $0.4 \%(\mathrm{w} / \mathrm{w})$ petroleum ether extract of Cloves flowering bud (Syzygium aromaticum) to the adults of some insects infesting stored maize grains inside the fiberglass bins at $32 \pm 2^{\circ} \mathrm{C}$ and $60 \pm 5 \% \mathrm{RH}$.

\begin{tabular}{|c|c|c|c|c|c|c|}
\hline \multirow[b]{2}{*}{ Insect species } & \multirow{2}{*}{$\begin{array}{c}\text { Exposure } \\
\text { period } \\
\text { (days) }\end{array}$} & \multicolumn{3}{|c|}{$\%$ adult mortality } & \multirow{2}{*}{$\begin{array}{l}\text { CO- } \\
\text { toxicity } \\
\text { factor }\end{array}$} & \multirow{2}{*}{$\begin{array}{c}\text { Type of } \\
\text { joint } \\
\text { action }\end{array}$} \\
\hline & & $\begin{array}{c}20 \pm 5 \% \mathrm{CO}_{2} \\
\text { alone } \\
\end{array}$ & $\begin{array}{c}0.4 \%(\mathrm{w} / \mathrm{w}) \\
\text { alone }\end{array}$ & $\begin{array}{c}\text { Extract }+ \\
\mathrm{CO}_{2}\end{array}$ & & \\
\hline \multirow{4}{*}{$\begin{array}{c}\text { Sitophilus } \\
\text { oryzae }\end{array}$} & 1 & $6.7 \pm 0.0$ & $37.4 \pm 1.9$ & $43.7 \pm 3.8$ & -0.9 & $d$ \\
\hline & 2 & $33.1 \pm 5.0$ & $98.7 \pm 1.9$ & $100 \pm 0.0$ & 0.0 & $d$ \\
\hline & 3 & $46.7 \pm 6.9$ & $100 \pm 0.0$ & $100 \pm 0.0$ & 0.0 & d \\
\hline & 5 & $52.7 \pm 3.3$ & $100 \pm 0.0$ & $100 \pm 0.0$ & 0.0 & $d$ \\
\hline Control & -- & $0.0 \pm 0.0$ & $0.0 \pm 0.0$ & $0.0 \pm 0.0$ & -- & -- \\
\hline \multirow{4}{*}{$\begin{array}{c}\text { Rhizopertha } \\
\text { dominica }\end{array}$} & 1 & $3.3 \pm 0.0$ & $73.8 \pm 1.9$ & $83.8 \pm 1.9$ & 8.7 & d \\
\hline & 2 & $6.1 \pm 1.9$ & $85.7 \pm 0.0$ & $97.5 \pm 2.0$ & 6.2 & d \\
\hline & 3 & $14.0 \pm 3.3$ & $85.7 \pm 0.0$ & $100 \pm 0.0$ & -0.3 & $d$ \\
\hline & 5 & $19.6 \pm 3.0$ & $85.7 \pm 0.0$ & $100 \pm 0.0$ & 0.0 & d \\
\hline Control & -- & $0.0 \pm 0.0$ & $0.0 \pm 0.0$ & $0.0 \pm 0.0$ & -- & -- \\
\hline \multirow{4}{*}{$\begin{array}{l}\text { Tribolium } \\
\text { castaneum }\end{array}$} & 1 & $3.3 \pm 0.0$ & $5.0 \pm 0.0$ & $8.3 \pm 1.9$ & 0.0 & d \\
\hline & 2 & $7.2 \pm 0.0$ & $8.3 \pm 1.9$ & $18.1 \pm 5.0$ & 16.7 & d \\
\hline & 3 & $16.8 \pm 1.9$ & $32.5 \pm 1.9$ & $42.1 \pm 3.3$ & -8.5 & d \\
\hline & 5 & $27.6 \pm 3.3$ & $51.7 \pm 2.0$ & $64.9 \pm 5.0$ & -18.2 & d \\
\hline Control & -- & $0.0 \pm 0.0$ & $0.0 \pm 0.0$ & $0.0 \pm 0.0$ & -- & -- \\
\hline
\end{tabular}

$\mathrm{d}=$ additive effect. 
Table 12. Joint action effect of $20 \pm 5 \% \mathrm{CO}_{2}$ and $0.8 \%(\mathrm{w} / \mathrm{w})$ petroleum ether extract of Cloves flowering bud (Syzygium aromaticum) to the adults of some insects infesting stored maize grains inside the fiberglass bins at $32 \pm 2^{\circ} \mathrm{C}$ and $60 \pm 5 \% \mathrm{RH}$.

\begin{tabular}{|c|c|c|c|c|c|c|}
\hline \multirow[b]{2}{*}{ Insect species } & \multirow[b]{2}{*}{$\begin{array}{c}\text { Exposure } \\
\text { period } \\
\text { (days) }\end{array}$} & \multicolumn{3}{|c|}{$\%$ adult mortality } & \multirow[b]{2}{*}{$\begin{array}{l}\text { CO- } \\
\text { toxicity } \\
\text { factor }\end{array}$} & \multirow[b]{2}{*}{$\begin{array}{c}\text { Type of } \\
\text { joint } \\
\text { action }\end{array}$} \\
\hline & & $\begin{array}{c}\mathrm{MA} \text { of } \\
20 \pm 5 \% \mathrm{CO}_{2} \\
\text { alone }\end{array}$ & $\begin{array}{c}\text { Extract at } \\
0.8 \%(\mathrm{w} / \mathrm{w}) \\
\text { alone }\end{array}$ & $\begin{array}{c}\text { Extract }+ \\
\mathrm{CO}_{2}\end{array}$ & & \\
\hline \multirow{4}{*}{$\begin{array}{c}\text { Sitophilus } \\
\text { oryzae }\end{array}$} & 1 & $6.7 \pm 0.0$ & $62.5 \pm 3.3$ & $73.7 \pm 3.3$ & 6.5 & d \\
\hline & 2 & $33.1 \pm 5.0$ & $100 \pm 0.0$ & $100 \pm 0.0$ & 0.0 & d \\
\hline & 3 & $46.7 \pm 6.9$ & $100 \pm 0.0$ & $100 \pm 0.0$ & 0.0 & $d$ \\
\hline & 5 & $52.7 \pm 3.3$ & $100 \pm 0.0$ & $100 \pm 0.0$ & 0.0 & d \\
\hline Control & -- & $0.0 \pm 0.0$ & $0.0 \pm 0.0$ & $0.0 \pm 0.0$ & -- & -- \\
\hline \multirow{4}{*}{$\begin{array}{c}\text { Rhizopertha } \\
\text { dominica }\end{array}$} & 1 & $3.3 \pm 0.0$ & $86.9 \pm 1.9$ & $97.5 \pm 2.0$ & 8.1 & $d$ \\
\hline & 2 & $6.1 \pm 1.9$ & $86.9 \pm 1.9$ & $97.5 \pm 3.8$ & 4.8 & $\mathrm{~d}$ \\
\hline & 3 & $14.0 \pm 3.3$ & $88.1 \pm 3.3$ & $100 \pm 0.0$ & 0.0 & d \\
\hline & 5 & $19.6 \pm 3.0$ & $89.2 \pm 2.0$ & $100 \pm 0.0$ & 0.0 & d \\
\hline Control & -- & $0.0 \pm 0.0$ & $0.0 \pm 0.0$ & $0.0 \pm 0.0$ & -- & -- \\
\hline \multirow{4}{*}{$\begin{array}{l}\text { Tribolium } \\
\text { castaneum }\end{array}$} & 1 & $3.3 \pm 0.0$ & $30.0 \pm 1.9$ & $34.9 \pm 3.3$ & 4.8 & d \\
\hline & 2 & $7.2 \pm 0.0$ & $36.1 \pm 5.0$ & $50.0 \pm 3.3$ & 15.5 & d \\
\hline & 3 & $16.8 \pm 1.9$ & $53.0 \pm 3.3$ & $63.8 \pm 3.8$ & -8.6 & d \\
\hline & 5 & $27.6 \pm 3.3$ & $81.8 \pm 3.3$ & $89.1 \pm 3.3$ & -10.9 & $\mathrm{~d}$ \\
\hline Control & -- & $0.0 \pm 0.0$ & $0.0 \pm 0.0$ & $0.0 \pm 0.0$ & -- & -- \\
\hline
\end{tabular}


Table 13. Joint action effect of $20 \pm 5 \% \mathrm{CO}_{2}$ and $0.4 \%(\mathrm{w} / \mathrm{w})$ acetone extract of White Mustard seeds (Brassica alba) to the adults of some insects infesting stored maize grains inside the fiberglass bins at $32 \pm 2^{\circ} \mathrm{C}$ and 60 $\pm 5 \% \mathrm{RH}$.

\begin{tabular}{|c|c|c|c|c|c|c|}
\hline \multirow[b]{2}{*}{ Insect species } & \multirow[b]{2}{*}{$\begin{array}{c}\text { Exposure } \\
\text { period } \\
\text { (days) }\end{array}$} & \multicolumn{3}{|c|}{$\%$ adult mortality } & \multirow[b]{2}{*}{$\begin{array}{l}\text { CO- } \\
\text { toxicity } \\
\text { factor }\end{array}$} & \multirow[b]{2}{*}{$\begin{array}{l}\text { Type of } \\
\text { joint } \\
\text { action }\end{array}$} \\
\hline & & $\begin{array}{c}\text { MA of } \\
20 \pm 5 \% \\
\mathrm{CO}_{2} \text { alone }\end{array}$ & $\begin{array}{c}\text { Extract at } \\
0.4 \%(\mathrm{w} / \mathrm{w}) \\
\text { alone }\end{array}$ & $\begin{array}{c}\text { Extract }+ \\
\mathrm{CO}_{2}\end{array}$ & & \\
\hline \multirow{4}{*}{$\begin{array}{c}\text { Sitophilus } \\
\text { oryzae }\end{array}$} & 1 & $6.7 \pm 0.0$ & $44.9 \pm 5.0$ & $57.4 \pm 1.9$ & 11.2 & $d$ \\
\hline & 2 & $33.1 \pm 5.0$ & $96.2 \pm 3.3$ & $100 \pm 0.0$ & 0.0 & d \\
\hline & 3 & $46.7 \pm 6.9$ & $97.4 \pm 3.8$ & $100 \pm 0.0$ & 0.0 & d \\
\hline & 5 & $52.7 \pm 3.3$ & $100 \pm 0.0$ & $100 \pm 0.0$ & 0.0 & $d$ \\
\hline Control & -- & $0.0 \pm 0.0$ & $0.0 \pm 0.0$ & $0.0 \pm 0.0$ & -- & -- \\
\hline \multirow{4}{*}{$\begin{array}{c}\text { Rhizopertha } \\
\text { dominica }\end{array}$} & 1 & $3.3 \pm 0.0$ & $7.4 \pm 0.0$ & $9.2 \pm 1.9$ & -14.0 & $d$ \\
\hline & 2 & $6.1 \pm 1.9$ & $14.9 \pm 1.9$ & $17.0 \pm 3.3$ & -19.0 & d \\
\hline & 3 & $14.0 \pm 3.3$ & $23.3 \pm 1.9$ & $30.0 \pm 3.3$ & -19.6 & d \\
\hline & 5 & $19.6 \pm 3.0$ & $34.5 \pm 1.9$ & $34.5 \pm 2.0$ & -19.6 & $d$ \\
\hline Control & -- & $0.0 \pm 0.0$ & $0.0 \pm 0.0$ & $0.0 \pm 0.0$ & -- & -- \\
\hline \multirow{4}{*}{$\begin{array}{l}\text { Tribolium } \\
\text { castaneum }\end{array}$} & 1 & $3.3 \pm 0.0$ & $3.3 \pm 0.0$ & $6.9 \pm 1.9$ & 4.5 & d \\
\hline & 2 & $7.2 \pm 0.0$ & $10.4 \pm 1.9$ & $14.0 \pm 1.9$ & -19.5 & d \\
\hline & 3 & $16.8 \pm 1.9$ & $14.0 \pm 1.9$ & $28.1 \pm 1.9$ & -8.8 & d \\
\hline & 5 & $27.6 \pm 3.3$ & $32.9 \pm 2.0$ & $48.9 \pm 3.3$ & -19.2 & d \\
\hline Control & -- & $0.0 \pm 0.0$ & $0.0 \pm 0.0$ & $0.0 \pm 0.0$ & -- & -- \\
\hline
\end{tabular}

$\mathrm{d}=$ additive effect. 
Table 14. Joint action effect of $20 \pm 5 \% \mathrm{CO}_{2}$ and $0.8 \%(\mathrm{w} / \mathrm{w})$ acetone extract of White Mustard seeds (Brassica alba) to the adults of some insects infesting stored maize grains inside the fiberglass bins at $32 \pm 2^{\circ} \mathrm{C}$ and 60 $\pm 5 \% \mathrm{RH}$.

\begin{tabular}{|c|c|c|c|c|c|c|}
\hline \multirow[b]{2}{*}{ Insect species } & \multirow[b]{2}{*}{$\begin{array}{c}\text { Exposure } \\
\text { period } \\
\text { (days) }\end{array}$} & \multicolumn{3}{|c|}{$\%$ adult mortality } & \multirow[b]{2}{*}{$\begin{array}{l}\text { CO- } \\
\text { toxicity } \\
\text { factor }\end{array}$} & \multirow[b]{2}{*}{$\begin{array}{c}\text { Type o } \\
\text { joint } \\
\text { action }\end{array}$} \\
\hline & & $\begin{array}{c}\text { MA of } \\
20 \pm 5 \% \\
\mathrm{CO}_{2} \text { alone }\end{array}$ & $\begin{array}{c}\text { Extract at } \\
0.8 \%(\mathrm{w} / \mathrm{w}) \\
\text { alone }\end{array}$ & $\begin{array}{c}\text { Extract + } \\
\mathrm{CO}_{2}\end{array}$ & & \\
\hline \multirow{4}{*}{$\begin{array}{c}\text { Sitophilus } \\
\text { oryzae }\end{array}$} & 1 & $6.7 \pm 0.0$ & $73.7 \pm 3.3$ & $75.0 \pm 1.9$ & -6.7 & d \\
\hline & 2 & $33.1 \pm 5.0$ & $100 \pm 0.0$ & $100 \pm 0.0$ & 0.0 & d \\
\hline & 3 & $46.7 \pm 6.9$ & $100 \pm 0.0$ & $100 \pm 0.0$ & 0.0 & d \\
\hline & 5 & $52.7 \pm 3.3$ & $100 \pm 0.0$ & $100 \pm 0.0$ & 0.0 & d \\
\hline Control & -- & $0.0 \pm 0.0$ & $0.0 \pm 0.0$ & $0.0 \pm 0.0$ & -- & -- \\
\hline \multirow{4}{*}{$\begin{array}{c}\text { Rhizopertha } \\
\text { dominica }\end{array}$} & 1 & $3.3 \pm 0.0$ & $13.0 \pm 2.0$ & $13.5 \pm 1.9$ & -17.2 & d \\
\hline & 2 & $6.1 \pm 1.9$ & $21.8 \pm 1.9$ & $24.6 \pm 1.9$ & -11.8 & $d$ \\
\hline & 3 & $14.0 \pm 3.3$ & $54.0 \pm 2.0$ & $61.6 \pm 1.9$ & -9.4 & d \\
\hline & 5 & $19.6 \pm 3.0$ & $70.1 \pm 1.9$ & $79.0 \pm 1.9$ & -11.9 & d \\
\hline Control & -- & $0.0 \pm 0.0$ & $0.0 \pm 0.0$ & $0.0 \pm 0.0$ & -- & -- \\
\hline \multirow{4}{*}{$\begin{array}{l}\text { Tribolium } \\
\text { castaneum }\end{array}$} & 1 & $3.3 \pm 0.0$ & $6.7 \pm 0.0$ & $12.0 \pm 1.9$ & 20.0 & d \\
\hline & 2 & $7.2 \pm 0.0$ & $16.7 \pm 1.9$ & $22.2 \pm 1.9$ & -7.1 & d \\
\hline & 3 & $16.8 \pm 1.9$ & $20.1 \pm 3.3$ & $44.0 \pm 5.0$ & 19.2 & d \\
\hline & 5 & $27.6 \pm 3.3$ & $57.6 \pm 3.3$ & $75.2 \pm 3.3$ & -11.7 & d \\
\hline Control & -- & $0.0 \pm 0.0$ & $0.0 \pm 0.0$ & $0.0 \pm 0.0$ & -- & -- \\
\hline
\end{tabular}


Table 15. Joint action effect of $20 \pm 5 \% \mathrm{CO}_{2}$ and $0.4 \%(\mathrm{w} / \mathrm{w})$ petroleum ether extract of White Mustard seeds (Brassica alba) to the adults of some insects infesting stored maize grains inside the fiberglass bins at $32 \pm 2{ }^{\circ} \mathrm{C}$ and 60 $\pm 5 \% \mathrm{RH}$.

\begin{tabular}{|c|c|c|c|c|c|c|}
\hline \multirow[b]{2}{*}{ Insect species } & \multirow{2}{*}{$\begin{array}{l}\text { Exposure } \\
\text { period } \\
\text { (days) }\end{array}$} & \multicolumn{3}{|c|}{$\%$ adult mortality } & \multirow{2}{*}{$\begin{array}{l}\text { CO- } \\
\text { toxicity } \\
\text { factor }\end{array}$} & \multirow{2}{*}{$\begin{array}{c}\text { Type of } \\
\text { joint } \\
\text { action }\end{array}$} \\
\hline & & $\begin{array}{c}20 \pm 5 \% \mathrm{CO}_{2} \\
\text { alone } \\
\end{array}$ & $\begin{array}{c}0.4 \%(\mathrm{w} / \mathrm{w}) \\
\text { alone }\end{array}$ & $\begin{array}{c}\text { Extract }+ \\
\mathrm{CO}_{2}\end{array}$ & & \\
\hline \multirow{4}{*}{$\begin{array}{c}\text { Sitophilus } \\
\text { oryzae }\end{array}$} & 1 & $6.7 \pm 0.0$ & $51.8 \pm 3.3$ & $63.0 \pm 1.9$ & 7.7 & $d$ \\
\hline & 2 & $33.1 \pm 5.0$ & $98.7 \pm 1.9$ & $100 \pm 0.0$ & 0.0 & d \\
\hline & 3 & $46.7 \pm 6.9$ & $100 \pm 0.0$ & $100 \pm 0.0$ & 0.0 & $d$ \\
\hline & 5 & $52.7 \pm 3.3$ & $100 \pm 0.0$ & $100 \pm 0.0$ & 0.0 & $d$ \\
\hline Control & -- & $0.0 \pm 0.0$ & $0.0 \pm 0.0$ & $0.0 \pm 0.0$ & -- & -- \\
\hline \multirow{4}{*}{$\begin{array}{c}\text { Rhizopertha } \\
\text { dominica }\end{array}$} & 1 & $3.3 \pm 0.0$ & $3.3 \pm 0.0$ & $6.0 \pm 0.0$ & -9.1 & d \\
\hline & 2 & $6.1 \pm 1.9$ & $13.0 \pm 1.9$ & $15.5 \pm 1.9$ & -18.8 & $d$ \\
\hline & 3 & $14.0 \pm 3.3$ & $34.5 \pm 1.9$ & $42.5 \pm 1.9$ & -12.4 & d \\
\hline & 5 & $19.6 \pm 3.0$ & $59.4 \pm 1.9$ & $63.7 \pm 1.9$ & -19.4 & d \\
\hline Control & -- & $0.0 \pm 0.0$ & $0.0 \pm 0.0$ & $0.0 \pm 0.0$ & -- & -- \\
\hline \multirow{4}{*}{$\begin{array}{l}\text { Tribolium } \\
\text { castaneum }\end{array}$} & 1 & $3.3 \pm 0.0$ & $3.3 \pm 0.0$ & $6.0 \pm 0.0$ & -9.1 & d \\
\hline & 2 & $7.2 \pm 0.0$ & $9.4 \pm 1.9$ & $15.0 \pm 1.9$ & -9.6 & $d$ \\
\hline & 3 & $16.8 \pm 1.9$ & $21.4 \pm 2.0$ & $32.1 \pm 3.3$ & -16.0 & d \\
\hline & 5 & $27.6 \pm 3.3$ & $32.0 \pm 3.3$ & $47.7 \pm 5.0$ & -20.0 & d \\
\hline Control & -- & $0.0 \pm 0.0$ & $0.0 \pm 0.0$ & $0.0 \pm 0.0$ & -- & -- \\
\hline
\end{tabular}

$\mathrm{d}=$ additive effect. 
Table 16. Joint action effect of $20 \pm 5 \% \mathrm{CO}_{2}$ and $0.8 \%(\mathrm{w} / \mathrm{w})$ petroleum ether extract of White Mustard seeds (Brassica alba) to the adults of some insects infesting stored maize grains inside the fiberglass bins at $32 \pm 2{ }^{\circ} \mathrm{C}$ and 60 $\pm 5 \% \mathrm{RH}$.

\begin{tabular}{|c|c|c|c|c|c|c|}
\hline \multirow[b]{2}{*}{ Insect species } & \multirow[b]{2}{*}{$\begin{array}{l}\text { Exposure } \\
\text { period } \\
\text { (days) }\end{array}$} & \multicolumn{3}{|c|}{$\%$ adult mortality } & \multirow[b]{2}{*}{$\begin{array}{l}\text { CO- } \\
\text { toxicity } \\
\text { factor }\end{array}$} & \multirow[b]{2}{*}{$\begin{array}{l}\text { Type of } \\
\text { joint } \\
\text { action }\end{array}$} \\
\hline & & $\begin{array}{c}\text { MA of } \\
20 \pm 5 \% \\
\mathrm{CO}_{2} \text { alone }\end{array}$ & $\begin{array}{c}\text { Extract at } \\
0.8 \% \\
(\mathrm{w} / \mathrm{w}) \\
\text { alone }\end{array}$ & $\begin{array}{c}\text { Extract + } \\
\mathrm{CO}_{2}\end{array}$ & & \\
\hline \multirow{4}{*}{$\begin{array}{c}\text { Sitophilus } \\
\text { oryzae }\end{array}$} & 1 & $6.7 \pm 0.0$ & $100 \pm 0.0$ & $96.7 \pm 0.0$ & -9.4 & $d$ \\
\hline & 2 & $33.1 \pm 5.0$ & $100 \pm 0.0$ & $100 \pm 0.0$ & 0.0 & $d$ \\
\hline & 3 & $46.7 \pm 6.9$ & $100 \pm 0.0$ & $100 \pm 0.0$ & 0.0 & $d$ \\
\hline & 5 & $52.7 \pm 3.3$ & $100 \pm 0.0$ & $100 \pm 0.0$ & 0.0 & $d$ \\
\hline Control & -- & $0.0 \pm 0.0$ & $0.0 \pm 0.0$ & $0.0 \pm 0.0$ & -- & -- \\
\hline \multirow{4}{*}{$\begin{array}{c}\text { Rhizopertha } \\
\text { dominica }\end{array}$} & 1 & $3.3 \pm 0.0$ & $65.4 \pm 1.9$ & $71.2 \pm 2.0$ & 3.6 & $d$ \\
\hline & 2 & $6.1 \pm 1.9$ & $65.4 \pm 1.9$ & $75.0 \pm 1.9$ & 4.9 & $d$ \\
\hline & 3 & $14.0 \pm 3.3$ & $79.7 \pm 1.9$ & $91.2 \pm 2.0$ & -2.7 & $d$ \\
\hline & 5 & $19.6 \pm 3.0$ & $84.4 \pm 3.3$ & $91.2 \pm 2.0$ & -8.8 & d \\
\hline Control & -- & $0.0 \pm 0.0$ & $0.0 \pm 0.0$ & $0.0 \pm 0.0$ & -- & -- \\
\hline \multirow{4}{*}{$\begin{array}{l}\text { Tribolium } \\
\text { castaneum }\end{array}$} & 1 & $3.3 \pm 0.0$ & $6.7 \pm 0.0$ & $8.0 \pm 1.9$ & -20.0 & $d$ \\
\hline & 2 & $7.2 \pm 0.0$ & $15.5 \pm 2.0$ & $21.4 \pm 1.9$ & -5.7 & $d$ \\
\hline & 3 & $16.8 \pm 1.9$ & $26.7 \pm 3.3$ & $43.9 \pm 3.8$ & 0.9 & $d$ \\
\hline & 5 & $27.6 \pm 3.3$ & $46.4 \pm 2.0$ & $60.6 \pm 3.8$ & -18.1 & $d$ \\
\hline Control & -- & $0.0 \pm 0.0$ & $0.0 \pm 0.0$ & $0.0 \pm 0.0$ & -- & -- \\
\hline
\end{tabular}

$\mathrm{d}=$ additive effect. 
Table 17. Joint action effect of $20 \pm 5 \% \mathrm{CO}_{2}$ and $0.4 \%(\mathrm{w} / \mathrm{w})$ acetone extract of Radish seeds (Raphanus sativus) to the adults of some insects infesting stored maize grains inside the fiberglass bins at $32 \pm 2^{\circ} \mathrm{C}$ and $60 \pm 5 \%$ $\mathrm{RH}$.

\begin{tabular}{|c|c|c|c|c|c|c|}
\hline \multirow[b]{2}{*}{ Insect species } & \multirow[b]{2}{*}{$\begin{array}{c}\text { Exposure } \\
\text { period } \\
\text { (days) }\end{array}$} & \multicolumn{3}{|c|}{$\%$ adult mortality } & \multirow[b]{2}{*}{$\begin{array}{l}\text { CO- } \\
\text { toxicity } \\
\text { factor }\end{array}$} & \multirow[b]{2}{*}{$\begin{array}{c}\text { Type of } \\
\text { joint } \\
\text { action }\end{array}$} \\
\hline & & $\begin{array}{c}\text { MA of } \\
20 \pm 5 \% \\
\mathrm{CO}_{2} \text { alone }\end{array}$ & $\begin{array}{c}\text { Extract at } \\
0.4 \% \\
(\mathrm{w} / \mathrm{w}) \\
\text { alone }\end{array}$ & $\begin{array}{c}\text { Extract + } \\
\mathrm{CO}_{2}\end{array}$ & & \\
\hline \multirow{4}{*}{$\begin{array}{c}\text { Sitophilus } \\
\text { oryzae }\end{array}$} & 1 & $6.7 \pm 0.0$ & $53.9 \pm 1.9$ & $56.2 \pm 5.0$ & -7.3 & $d$ \\
\hline & 2 & $33.1 \pm 5.0$ & $91.2 \pm 2.0$ & $100 \pm 0.0$ & 0.0 & $d$ \\
\hline & 3 & $46.7 \pm 6.9$ & $100 \pm 0.0$ & $100 \pm 0.0$ & 0.0 & $d$ \\
\hline & 5 & $52.7 \pm 3.3$ & $100 \pm 0.0$ & $100 \pm 0.0$ & 0.0 & $d$ \\
\hline Control & -- & $0.0 \pm 0.0$ & $0.0 \pm 0.0$ & $0.0 \pm 0.0$ & -- & -- \\
\hline \multirow{4}{*}{$\begin{array}{c}\text { Rhizopertha } \\
\text { dominica }\end{array}$} & 1 & $3.3 \pm 0.0$ & $3.3 \pm 0.0$ & $6.0 \pm 0.0$ & -9.1 & $d$ \\
\hline & 2 & $6.1 \pm 1.9$ & $10.1 \pm 1.9$ & $13.0 \pm 0.0$ & -19.7 & $d$ \\
\hline & 3 & $14.0 \pm 3.3$ & $57.6 \pm 3.3$ & $67.0 \pm 1.9$ & -6.4 & $d$ \\
\hline & 5 & $19.6 \pm 3.0$ & $82.2 \pm 1.9$ & $89.4 \pm 0.0$ & -10.6 & $d$ \\
\hline Control & -- & $0.0 \pm 0.0$ & $0.0 \pm 0.0$ & $0.0 \pm 0.0$ & -- & -- \\
\hline \multirow{4}{*}{$\begin{array}{l}\text { Tribolium } \\
\text { castaneum }\end{array}$} & 1 & $3.3 \pm 0.0$ & $0.0 \pm 0.0$ & $3.3 \pm 0.0$ & 0.0 & d \\
\hline & 2 & $7.2 \pm 0.0$ & $10.0 \pm 0.0$ & $14.0 \pm 0.0$ & -18.6 & $d$ \\
\hline & 3 & $16.8 \pm 1.9$ & $21.4 \pm 3.3$ & $31.0 \pm 1.9$ & -18.8 & d \\
\hline & 5 & $27.6 \pm 3.3$ & $23.0 \pm 2.0$ & $41.0 \pm 1.9$ & -19.0 & d \\
\hline Control & -- & $0.0 \pm 0.0$ & $0.0 \pm 0.0$ & $0.0 \pm 0.0$ & -- & -- \\
\hline
\end{tabular}

$\mathrm{d}=$ additive effect. 
Table 18. Joint action effect of $20 \pm 5 \% \mathrm{CO}_{2}$ and $0.8 \%(\mathrm{w} / \mathrm{w})$ acetone extract of Radish seeds (Raphanus sativus) to the adults of some insects infesting stored maize grains inside the fiberglass bins at $32 \pm 2{ }^{\circ} \mathrm{C}$ and $60 \pm 5 \%$ $\mathrm{RH}$.

\begin{tabular}{|c|c|c|c|c|c|c|}
\hline \multirow[b]{2}{*}{ Insect species } & \multirow[b]{2}{*}{$\begin{array}{l}\text { Exposure } \\
\text { period } \\
\text { (days) }\end{array}$} & \multicolumn{3}{|c|}{$\%$ adult mortality } & \multirow[b]{2}{*}{$\begin{array}{l}\text { CO- } \\
\text { toxicity } \\
\text { factor }\end{array}$} & \multirow[b]{2}{*}{$\begin{array}{c}\text { Type of } \\
\text { joint } \\
\text { action }\end{array}$} \\
\hline & & $\begin{array}{c}\text { MA of } \\
20 \pm 5 \% \\
\mathrm{CO}_{2} \text { alone }\end{array}$ & $\begin{array}{c}\text { Extract at } \\
0.8 \% \\
(\mathrm{w} / \mathrm{w}) \\
\text { alone }\end{array}$ & $\begin{array}{c}\text { Extract }+ \\
\mathrm{CO}_{2}\end{array}$ & & \\
\hline \multirow{4}{*}{$\begin{array}{c}\text { Sitophilus } \\
\text { oryzae }\end{array}$} & 1 & $6.7 \pm 0.0$ & $86.2 \pm 3.3$ & $89.9 \pm 5.0$ & -3.2 & d \\
\hline & 2 & $33.1 \pm 5.0$ & $100 \pm 0.0$ & $100 \pm 0.0$ & 0.0 & d \\
\hline & 3 & $46.7 \pm 6.9$ & $100 \pm 0.0$ & $100 \pm 0.0$ & 0.0 & $d$ \\
\hline & 5 & $52.7 \pm 3.3$ & $100 \pm 0.0$ & $100 \pm 0.0$ & 0.0 & $d$ \\
\hline Control & -- & $0.0 \pm 0.0$ & $0.0 \pm 0.0$ & $0.0 \pm 0.0$ & -- & -- \\
\hline \multirow{4}{*}{$\begin{array}{c}\text { Rhizopertha } \\
\text { dominica }\end{array}$} & 1 & $3.3 \pm 0.0$ & $55.1 \pm 5.0$ & $62.0 \pm 6.6$ & 6.2 & d \\
\hline & 2 & $6.1 \pm 1.9$ & $78.8 \pm 3.3$ & $88.6 \pm 3.3$ & 4.4 & d \\
\hline & 3 & $14.0 \pm 3.3$ & $88.2 \pm 1.9$ & $91.1 \pm 1.9$ & -8.9 & d \\
\hline & 5 & $19.6 \pm 3.0$ & $91.1 \pm 1.9$ & $94.0 \pm 1.9$ & -6.0 & $d$ \\
\hline Control & -- & $0.0 \pm 0.0$ & $0.0 \pm 0.0$ & $0.0 \pm 0.0$ & -- & -- \\
\hline \multirow{4}{*}{$\begin{array}{l}\text { Tribolium } \\
\text { castaneum }\end{array}$} & 1 & $3.3 \pm 0.0$ & $5.6 \pm 0.0$ & $10.5 \pm 1.9$ & 18.0 & d \\
\hline & 2 & $7.2 \pm 0.0$ & $13.0 \pm 1.9$ & $18.0 \pm 1.9$ & -10.9 & d \\
\hline & 3 & $16.8 \pm 1.9$ & $24.9 \pm 3.3$ & $34.0 \pm 3.8$ & -18.5 & d \\
\hline & 5 & $27.6 \pm 3.3$ & $30.0 \pm 3.3$ & $47.0 \pm 1.9$ & -18.4 & d \\
\hline Control & -- & $0.0 \pm 0.0$ & $0.0 \pm 0.0$ & $0.0 \pm 0.0$ & -- & -- \\
\hline
\end{tabular}

$\mathrm{d}=$ additive effect. 
Table 19. Joint action effect of $20 \pm 5 \% \mathrm{CO}_{2}$ and $0.4 \%(\mathrm{w} / \mathrm{w})$ Petroleum ether extract of Radish seeds (Raphanus sativus) to the adults of some insects infesting stored maize grains inside the fiberglass bins at $32 \pm 2^{\circ} \mathrm{C}$ and $60 \pm 5 \%$ $\mathrm{RH}$.

\begin{tabular}{|c|c|c|c|c|c|c|}
\hline \multirow[b]{2}{*}{ Insect species } & \multirow[b]{2}{*}{$\begin{array}{c}\text { Exposure } \\
\text { period } \\
\text { (days) }\end{array}$} & \multicolumn{3}{|c|}{$\%$ adult mortality } & \multirow[b]{2}{*}{$\begin{array}{l}\text { CO-toxicity } \\
\text { factor }\end{array}$} & \multirow[b]{2}{*}{$\begin{array}{c}\text { Type of } \\
\text { joint } \\
\text { action }\end{array}$} \\
\hline & & $\begin{array}{c}\text { MA of } \\
20 \pm 5 \% \mathrm{CO}_{2} \\
\text { alone }\end{array}$ & $\begin{array}{c}\text { Extract at } \\
0.4 \%(\mathrm{w} / \mathrm{w}) \\
\text { alone }\end{array}$ & $\begin{array}{c}\text { Extract }+ \\
\mathrm{CO}_{2}\end{array}$ & & \\
\hline \multirow{4}{*}{ Sitophilus oryzae } & 1 & $6.7 \pm 0.0$ & $44.9 \pm 1.9$ & $60.0 \pm 1.9$ & 16.3 & $d$ \\
\hline & 2 & $33.1 \pm 5.0$ & $100 \pm 0.0$ & $100 \pm 0.0$ & 0.0 & d \\
\hline & 3 & $46.7 \pm 6.9$ & $100 \pm 0.0$ & $100 \pm 0.0$ & 0.0 & $d$ \\
\hline & 5 & $52.7 \pm 3.3$ & $100 \pm 0.0$ & $100 \pm 0.0$ & 0.0 & d \\
\hline Control & -- & $0.0 \pm 0.0$ & $0.0 \pm 0.0$ & $0.0 \pm 0.0$ & -- & -- \\
\hline \multirow{4}{*}{$\begin{array}{c}\text { Rhizopertha } \\
\text { dominica }\end{array}$} & 1 & $3.3 \pm 0.0$ & $29.6 \pm 1.9$ & $29.6 \pm 3.3$ & -10.0 & $d$ \\
\hline & 2 & $6.1 \pm 1.9$ & $32.1 \pm 0.0$ & $33.3 \pm 3.3$ & -12.8 & d \\
\hline & 3 & $14.0 \pm 3.3$ & $35.6 \pm 3.3$ & $44.4 \pm 0.0$ & -10.5 & d \\
\hline & 5 & $19.6 \pm 3.0$ & $54.6 \pm 5.0$ & $63.0 \pm 3.3$ & -15.1 & d \\
\hline Control & -- & $0.0 \pm 0.0$ & $0.0 \pm 0.0$ & $0.0 \pm 0.0$ & -- & -- \\
\hline \multirow{4}{*}{$\begin{array}{l}\text { Tribolium } \\
\text { castaneum }\end{array}$} & 1 & $3.3 \pm 0.0$ & $3.3 \pm 0.0$ & $6.0 \pm 0.0$ & -9.0 & $d$ \\
\hline & 2 & $7.2 \pm 0.0$ & $8.3 \pm 0.0$ & $18.0 \pm 0.0$ & 16.1 & $d$ \\
\hline & 3 & $16.8 \pm 1.9$ & $10.0 \pm 1.9$ & $29.2 \pm 3.3$ & 9.0 & d \\
\hline & 5 & $27.6 \pm 3.3$ & $27.6 \pm 1.9$ & $45.0 \pm 3.3$ & -18.5 & d \\
\hline Control & -- & $0.0 \pm 0.0$ & $0.0 \pm 0.0$ & $0.0 \pm 0.0$ & -- & -- \\
\hline
\end{tabular}

$\mathrm{d}=$ additive effect. 
Table 20. Joint action effect of $20 \pm 5 \% \mathrm{CO}_{2}$ and $0.8 \%(\mathrm{w} / \mathrm{w})$ Petroleum ether extract of Radish seeds (Raphanus sativus) to the adults of some insects infesting stored maize grains inside the fiberglass bins at $32 \pm 2{ }^{\circ} \mathrm{C}$ and $60 \pm 5 \%$ $\mathrm{RH}$.

\begin{tabular}{|c|c|c|c|c|c|c|}
\hline \multirow[b]{2}{*}{ Insect species } & \multirow[b]{2}{*}{$\begin{array}{l}\text { Exposure } \\
\text { period } \\
\text { (days) }\end{array}$} & \multicolumn{3}{|c|}{$\%$ adult mortality } & \multirow[b]{2}{*}{$\begin{array}{l}\text { CO- } \\
\text { toxicity } \\
\text { factor }\end{array}$} & \multirow[b]{2}{*}{$\begin{array}{l}\text { Type of } \\
\text { joint } \\
\text { action }\end{array}$} \\
\hline & & $\begin{array}{c}\text { MA of } \\
20 \pm 5 \% \\
\mathrm{CO}_{2} \text { alone }\end{array}$ & $\begin{array}{c}\text { Extract at } \\
0.8 \% \\
(\mathrm{w} / \mathrm{w}) \\
\text { alone }\end{array}$ & $\begin{array}{c}\text { Extract + } \\
\mathrm{CO}_{2}\end{array}$ & & \\
\hline \multirow{4}{*}{$\begin{array}{c}\text { Sitophilus } \\
\text { oryzae }\end{array}$} & 1 & $6.7 \pm 0.0$ & $72.5 \pm 1.9$ & $95.0 \pm 1.9$ & 19.9 & $d$ \\
\hline & 2 & $33.1 \pm 5.0$ & $100 \pm 0.0$ & $100 \pm 0.0$ & 0.0 & $d$ \\
\hline & 3 & $46.7 \pm 6.9$ & $100 \pm 0.0$ & $100 \pm 0.0$ & 0.0 & $d$ \\
\hline & 5 & $52.7 \pm 3.3$ & $100 \pm 0.0$ & $100 \pm 0.0$ & 0.0 & $d$ \\
\hline Control & -- & $0.0 \pm 0.0$ & $0.0 \pm 0.0$ & $0.0 \pm 0.0$ & -- & -- \\
\hline \multirow{4}{*}{$\begin{array}{c}\text { Rhizopertha } \\
\text { dominica }\end{array}$} & 1 & $3.3 \pm 0.0$ & $78.5 \pm 3.3$ & $88.8 \pm 3.3$ & 8.6 & $d$ \\
\hline & 2 & $6.1 \pm 1.9$ & $85.7 \pm 0.0$ & $96.7 \pm 0.0$ & 5.3 & $d$ \\
\hline & 3 & $14.0 \pm 3.3$ & $88.1 \pm 1.9$ & $96.7 \pm 0.0$ & -3.3 & $d$ \\
\hline & 5 & $19.6 \pm 3.0$ & $95.1 \pm 1.9$ & $96.7 \pm 0.0$ & -3.3 & d \\
\hline Control & -- & $0.0 \pm 0.0$ & $0.0 \pm 0.0$ & $0.0 \pm 0.0$ & -- & -- \\
\hline \multirow{4}{*}{$\begin{array}{l}\text { Tribolium } \\
\text { castaneum }\end{array}$} & 1 & $3.3 \pm 0.0$ & $12.0 \pm 1.9$ & $16.7 \pm 1.9$ & 9.2 & $d$ \\
\hline & 2 & $7.2 \pm 0.0$ & $20.4 \pm 3.3$ & $26.7 \pm 3.3$ & -3.3 & $d$ \\
\hline & 3 & $16.8 \pm 1.9$ & $27.8 \pm 1.9$ & $37.5 \pm 1.9$ & -15.9 & $d$ \\
\hline & 5 & $27.6 \pm 3.3$ & $53.0 \pm 6.6$ & $82.2 \pm 5.0$ & 2.0 & $d$ \\
\hline Control & -- & $0.0 \pm 0.0$ & $0.0 \pm 0.0$ & $0.0 \pm 0.0$ & -- & -- \\
\hline
\end{tabular}

$\mathrm{d}=$ additive effect. 


\section{REFERENCES}

1. Abbott, W. W. 1925. A method computing the effectiveness of an insecticide. J. Econ. Entomol., 18: 265-267.

2. Abd El-Aziz, A. E. 2002. Effectiveness of petroleum ether extract of some plants alone and under controlled atmospheres of carbon dioxide against the cowpea beetle Callosobruchus maculatus (F.). Annals of Agric. Sci. Moshtohor, 40 (2): 1309-1319.

3. Aliniazee, M. T. 1971. The effect of carbon dioxide gas alone or in combinations on the mortality of Tribolium castaneum (Herbst.) and T. confusum (Duval.), Coleoptera, Tenebrionidae. J. Stored Prod. Res., 7: 243-252.

4. Bansley, E. A. 1968. Teuahedrol, 24, 3747. (Cited from Jeffery et al., 1996).

5. Cronwell, N. H. 1979. Chem. Rev., 79, 331 (Cited from fery et al., 1996).

6. El-Lakwah, F. A., O.M. Khaled and Z. A. Halawa. 1996. Effect of modified atmosphere of more than $99 \%$ nitrogen or enriched with carbon dioxide on mortality of some stored product insects, seed germination, chlorophyll and carotene contents of certain crop seedlings. Annals of Agric. Sci., Moshtohor, 34 (4): 1869-1877.

7. El-Lakwah, F. A., Sanaa M. Mahgoub and Salwa M. Mohamed. 2000. Effect of maize husk ash and mustard seeds powder (Brassica arvesis) as grains protectants on some stored product insects. Annals of Agric. Sci., Moshtohor, 38 (1): 565-571.

8. Halaw, Z. A.,I.A.EL- Sappagh and M. M. EL-Assal 2009. Toxicity of Tooth pick Seed ( Ammi visnaga L.) extracts against the lesser grain borer, Rhizopertha dominica, F. (Bostrychidae: Coloeoptera) Egypt. J.Agric. Res. 88(2) 485-493.

9. Halawa, Z. A. 2003. An attempt to reduce $\mathrm{pH} 3$ dose as a fumigant using a mixture with $\mathrm{CO}_{2}$ against stored product mites in Egypt. The $2^{\text {nd }}$ Conference of Agricultural and Biological Research Division "Prospects of the Resent Agricultural Research". Euunt. J. Agric. Res. NRC-1 (3): 631-644.

10. Halawa, Z. A., R. A. Mohamed and I. H. El-Kashlan. 1998. Laboratory evaluation of some plants and insecticides against cowpea beetle (Callosobruchus maculates, F.) infesting stored products. Egypt. J. Agric. Res., 76 (1): 85-94.

11. Harein, P. K. and A.E. Press. 1968. Mortality of stored peanut insects exposed to mixtures of atmospheric gases at various temperatures. J. Stored Prod. Res., 4: 77-82. 
12. Ivbijaro, M. F. 1984. Toxic effects of groundnut oil on the rice weevil Sitophilus oryzae. Insect Sci., Appl., 5: 251-252.

13. Jay, E. G. and G. C. Pearman. 1971. Susceptibility of two species of Tribolium (Coleoptera, Tenebrionidae) to alternations of atmospheric gas concentrations. J. Stored Prod. Res., 7: 181-188.

14. Keethaas, D. R. 1931. Biochem. 230, 440 (Cited from jeffery et al., 1996).

15. Krishnamurthy, T.S., E.C. Speatt and C.L. Bell. 1986. The toxicity of carbon dioxide to adult beetles in low oxygen atmospheres. Journal-of-Stored-ProductsResearch. 22: 3, 145-151.

16. Mansor, N. A., M. E. Defrawi , A. Toppozada and M. Zeid. 1966. Toxicological studies on the Egyptian cotton leaf work, Prodenia litura. Potentiation and antagonism of organophosphorus and carbamate insecticide. J. Econ. Ent., 59: 307-311.

17. Mohamed, R. A. 1999. Effectiveness of some plant extracts under modified atmospheres against the cowpea beetle (Callosobruchus maculatus, F.). Annals of Agric. Sci. Moshtohor, 37 (1): 709-720.

18. Mohandas, J. 1969. Aust. J. Chem., 22, 1803. (Cited from jeffery et. al., 1996).

19. Okuda, T. J. 1983 . Chem. Soc., Perkin Trans. 1, 1765. (Cited from jeffery et. al., 1996).

20. Reichmuth, C. 1986. Low oxygen content to control stored product insects. In Donahaye, E. and Navarro, S. ed., Proceedings of the $4^{\text {th }}$. International working conference on stored product protection. Tel. Aviv, Israel Sept./1986, 194-207.

21. Sendecor, G. W. and W. G. Cochran. 1989. Statistical methods. $8^{\text {th }}$ ed., Iowa State Univ., M Press, Iowa, U.S.A.

22. Shimizu, Y. J. 1986. Am. Chem. Soc., 108, 514 (Cited from jeffery et al., 1996).

23. Su, H. C. F. 1985. Laboratory evaluation of biological activity of Cinnamomum cassia to four species of stored product insects. J. Ent. Sci., 20: 2, 247-253.

24. Tunc, I. 1983. Mortality of Tribolium confusum Duval. (Tenebriondae, Coleoptera) adults in various atmospheric gases compositions. Zeitschrift für Angewandts Entomologie, 95 (3): 263-264. 


\title{
التأثثر المشترك لبعض مخاليط المستخلصات النباتية مع جو معدل من ثانى أكسيد الكربون ضد حشرات المواد المخزونة
}

\author{
فارس أمين اللقوة ، ، زغلول عبد الفتاح حلاوةّ، متولى مصطفى خطاب 1 ،

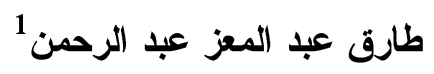 \\ ا ـ قسم وقاية النبات - كلية الزراعة بشتنهر - جامعة بنها.

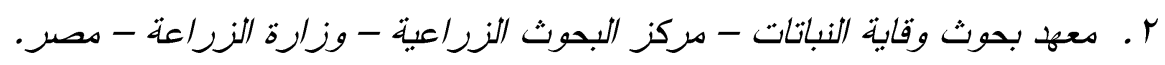

أجريت هذه التجارب داخل الصوامع الفيبرجلاس أثناء فصل الصيف وكانت درجة حرارة

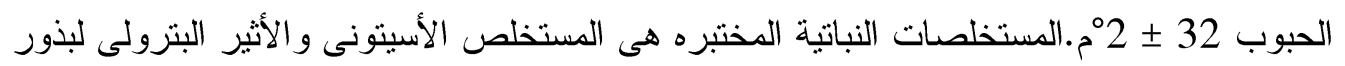

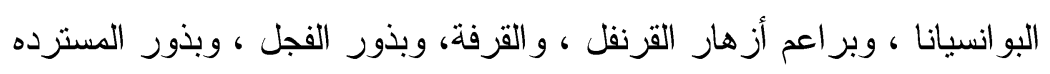

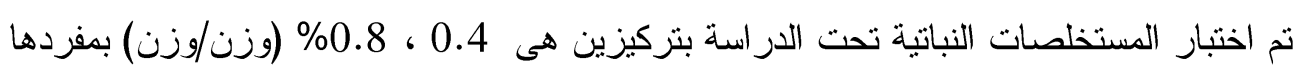
وتحت جو معدل يحتوى على 20 × 5\% ثانى أكسيد الكربون ضد الحشر ات اتركئ الكاملة لكل من سوسة الأرز ، وثاقبة الحبوب الصغرى ، وخنفساء الدقيق الكستنئيائية.

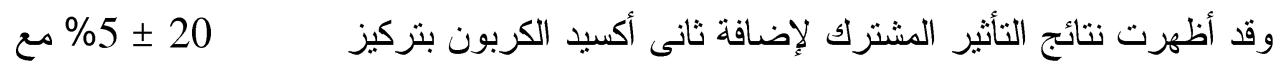

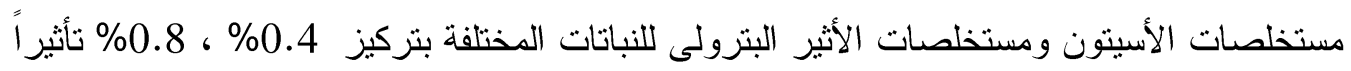

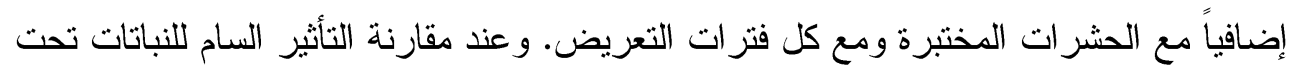

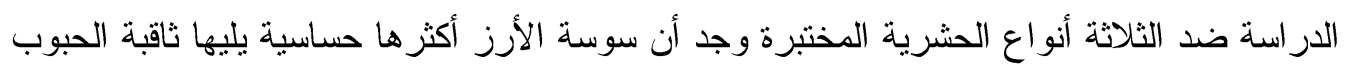

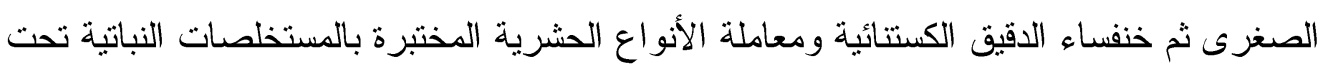

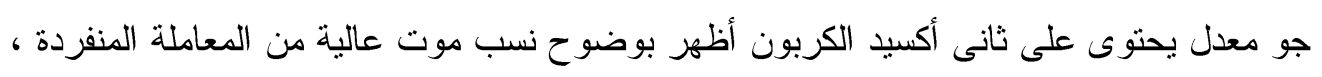

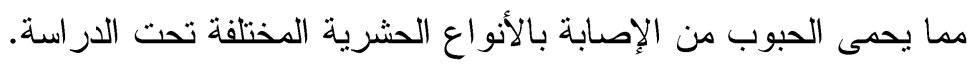

